\title{
On the Existence of Mixed Strategy Nash Equilibria
}

\author{
Pavlo Prokopovych ${ }^{\mathrm{a}, 1}$ and Nicholas C. Yannelis ${ }^{\mathrm{b}}$ \\ ${ }^{a}$ Kyiv School of Economics, 1 Ivana Mazepy, Kyiv 01010, Ukraine \\ ${ }^{\mathrm{b}}$ Department of Economics, Tippie College of Business, University of Iowa, \\ Iowa City, IA 52242-1994, USA
}

\begin{abstract}
The focus of this paper is on developing verifiable sufficient conditions for the existence of a mixed strategy Nash equilibrium for both diagonally transfer continuous and better-reply secure games. First, we show that employing the concept of diagonal transfer continuity in place of betterreply security might be advantageous when the existence of a mixed strategy Nash equilibrium is concerned. Then, we study equilibrium existence in better-reply secure games possessing a payoff secure mixed extension. With the aid of an example, we show that such games need not have mixed strategy Nash equilibria. We provide geometric conditions for the mixed extension of a two-person game that is reciprocally upper semicontinuous and uniformly payoff secure to be better-reply secure.
\end{abstract}

Keywords Discontinuous game; Diagonally transfer continuous game; Better-reply secure game; Mixed strategy equilibrium; Transfer lower semicontinuity

\section{JEL classification Numbers C65; C72}

Date April 18, 2014

\footnotetext{
${ }^{1}$ Corresponding author. E-mail address: pprokopo@gmail.com, Phone: +38-044-4928012, Fax: +38-044-492-8011
} 


\section{Introduction}

Nowadays, one of the main tools in the arsenal of economists concerned with equilibrium existence is Reny's (1999) theorem, according to which a compact Borel game has a mixed strategy Nash equilibrium if its mixed extension is better-reply secure. $^{2}$ In applications, better-reply security usually follows from two conditions: one related to reciprocal upper semicontinuity and the other to payoff security.

Establishing the payoff security of a game's mixed extension often constitutes a complicated problem. The concept of uniform payoff security, introduced by Monteiro and Page (2007), makes the problem considerably more tractable in games where it is applicable, including catalog games (Page and Monteiro, 2003) and voting models (Carbonell-Nicolau and Ok, 2007). ${ }^{3}$ Verifying whether a game that is not upper semicontinuous-sum has a betterreply secure mixed extension is, as a rule, quite challenging. This paper's main focus is on studying the existence of a mixed strategy Nash equilibrium in normal form games where the sum of the payoff functions is not necessarily upper semicontinuous.

We begin with considering the games having a diagonally transfer continuous mixed extension, appealing to the analogy with the better-reply secure mixed extensions. Baye, Tian, and Zhou (1993) showed that the existence of a pure strategy Nash equilibrium in diagonally transfer continuous games follows from a generalization of the Knuster-Kuratowski-Mazurkiewicz (KKM) lemma. ${ }^{4}$ In Section 2 of this paper, the Ky Fan minimax inequality, in a slightly generalized form, is used to prove that every compact Borel game

\footnotetext{
${ }^{2} \mathrm{~A}$ number of results extending Reny's equilibrium existence theorem have been obtained recently (see, e.g., Barelli and Meneghel, 2013; Bich, 2009; Carmona, 2011; de Castro, 2011; McLennan, Monteiro, and Tourky, 2011; Reny, 2013)

${ }^{3}$ Another approach to showing the payoff security of mixed extensions can be found in Duggan (2007), where hospitable strategies are used for studying equilibrium existence in voting models.

${ }^{4}$ The first part of this paper contains a number of results first presented in our 2012 working paper "On Uniform Conditions for the Existence of Mixed Strategy Equilibria."
} 
whose mixed extension is diagonally transfer continuous has a mixed strategy Nash equilibrium. The range of applications of this basic result is considerably broader than that of Glicksberg's (1952) equilibrium existence theorem - whose proof is based on the Kakutani-Fan-Glicksberg fixed point theorem. In particular, the mixed extension of a game is diagonally transfer continuous if the following two conventional assumptions hold: the extension is payoff secure and the game is upper semicontinuous-sum. Then, in Section 3, we extend the concept of uniform payoff security to diagonally transfer continuous games by introducing uniform diagonal security. In the upper semicontinuous-sum games, uniform payoff security implies uniform diagonal security. At the same time, if a compact Borel game is uniformly diagonally secure, it has a mixed strategy Nash equilibrium, which makes it possible to avoid having to study any additional properties of the game's mixed extension.

Example 1 is a slight modification of the Tullock rent-seeking game where it is additionally assumed that the favor the players vie for is granted to a third party with probability one-half if at least one player exerts no effort at all. Notwithstanding the fact that the game is not better-reply secure, it is not only diagonally transfer continuous, but also uniformly diagonally secure; that is, the game has a mixed strategy Nash equilibrium.

In Section 4, we adapt Simon's (1987) concept of weak domination on average to our setting by introducing weak uniform payoff security, a generalization of uniform payoff security. Using this concept, we construct a better-reply secure two-person game with a payoff secure mixed extension that has no mixed strategy equilibria (Example 2).

In Section 5, we study the existence of a mixed strategy equilibrium in reciprocally upper semicontinuous games that are uniformly payoff secure. In such games, the equilibrium existence problem becomes considerably more tractable if it is possible to transform the game into an upper semicontinuous- 
sum game with the aid of positive affine transformations, ${ }^{5}$ which, in particular, implies that the game also has a reciprocally upper semicontinuous mixed extension. In Example 3, this technique is applied to a conventional two-candidate probabilistic spatial voting game. Theorems 5 and 6 give geometric sufficient conditions for games that are reciprocally upper semicontinuous and uniformly payoff secure to have a better-reply secure mixed extension.

The Appendix contains a number of auxiliary results, deferred proofs, and some comments regarding Theorem 5b of Dasgupta and Maskin (1986).

\section{The Model and Some Facts}

We consider a game $G=\left(X_{i}, u_{i}\right)_{i \in I}$, where $I=\{1, \ldots, n\}$, each player $i$ 's pure strategy set $X_{i}$ is a nonempty, compact subset of a metrizable topological vector space, and each payoff function $u_{i}$ is a bounded Borel measurable function from the Cartesian product $X=\prod_{i \in I} X_{i}$, equipped with the product topology, to $\mathbb{R}$. Under these conditions, $G=\left(X_{i}, u_{i}\right)_{i \in I}$ is called a compact Borel game. A game $G=\left(X_{i}, u_{i}\right)_{i \in I}$ is quasiconcave if each $X_{i}$ is convex and $u_{i}\left(\cdot, x_{-i}\right): X_{i} \rightarrow \mathbb{R}$ is quasiconcave for all $i \in I$ and all $x_{-i} \in X_{-i}$, where $X_{-i}=\Pi_{k \in I \backslash\{i\}} X_{k}$. In this paper, by a game we mean a compact Borel game.

The following definition of a payoff secure game is due to Reny (1999).

Definition 1 In $G=\left(X_{i}, u_{i}\right)_{i \in I}$, player $i$ can secure a payoff of $\alpha \in \mathbb{R}$ at $x \in X$ if there exists $d_{i} \in X_{i}$ such that $u_{i}\left(d_{i}, x_{-i}^{\prime}\right) \geq \alpha$ for all $x_{-i}^{\prime}$ in some open neighborhood of $x_{-i}$. The game $G$ is payoff secure if for every $x \in X$ and every $\varepsilon>0$, each player $i$ can secure a payoff of $u_{i}(x)-\varepsilon$ at $x$.

Payoff security can be reformulated in terms of transfer lower semicontinuity, due to Tian (1992).

\footnotetext{
${ }^{5}$ Using a similar approach, Amir (2005) gives examples of Cournot oligopolies possessing the cardinal complementarity property where the other complementarity conditions are ineffective.
} 
Definition 2 Let $Z$ and $Y$ be two topological spaces. A function $f: Z \times$ $Y \rightarrow \mathbb{R}$ is $\lambda$-transfer lower semicontinuous in $y$ if for every $(z, y) \in Z \times Y$, $f(z, y)>\lambda$ implies that there exists some point $z^{\prime} \in Z$ and some neighborhood $\mathcal{N}_{Y}(y)$ of $y$ in $Y$ such that $f\left(z^{\prime}, w\right)>\lambda$ for all $w \in \mathcal{N}_{Y}(y)$. A function $f: Z \times Y \rightarrow \mathbb{R}$ is transfer lower semicontinuous in $y$ if $f$ is $\lambda$-transfer lower semicontinuous in $y$ for every $\lambda \in \mathbb{R}$.

A game is payoff secure if and only if each player's payoff function is transfer lower semicontinuous in the other players' strategies (see Prokopovych, 2011, Lemma 1).

The graph of $G$ is defined by $\operatorname{Gr} G=\left\{(x, u) \in X \times \mathbb{R}^{n} \mid u_{i}(x)=u_{i}\right.$ for all $i \in N\}$, and the set of pure strategy Nash equilibria of $G$ in $X$ is denoted by $E_{G}$. For a subset $B$ of a topological vector space $X$, we denote by $\operatorname{cl} B$ the closure of $B$ and by $\operatorname{co} B$ the convex hull of $B$. In a metric space $Y$, we denote by $B_{Y}(y, r)$ the open ball centered at $y$ and with radius $r>0$.

Definition 3 A game $G=\left(X_{i}, u_{i}\right)_{i \in I}$ is better-reply secure if whenever $\left(x^{*}, u^{*}\right) \in \operatorname{clGr} G$ and $x^{*} \in X \backslash E_{G}$, some player $i$ can secure a payoff strictly above $u_{i}^{*}$ at $x^{*}$.

A useful fact is that a payoff secure game is better-reply secure iff it is also transfer reciprocally upper semicontinuous (see Bagh and Jofre, 2006; Prokopovych, 2011, Lemma 2).

Definition 4 A game $G=\left(X_{i}, u_{i}\right)_{i \in I}$ is: (i) reciprocally upper semicontinuous if for any $(x, \alpha) \in \operatorname{clGr} G \backslash \operatorname{Gr} G$, there is a player $i$ such that $u_{i}(x)>\alpha_{i}$; (ii) weakly reciprocally upper semicontinuous if whenever $(x, \alpha) \in \operatorname{clGr} G \backslash \operatorname{Gr} G$, there are a player $i$ and $d_{i} \in X_{i}$ such that $u_{i}\left(d_{i}, x_{-i}\right)>\alpha_{i}$; (iii) transfer reciprocally upper semicontinuous if whenever $(x, \alpha) \in \operatorname{clGr} G \backslash \operatorname{Gr} G$ and $x$ is not a Nash equilibrium, there are a player $i$ and $d_{i} \in X_{i}$ such that $u_{i}\left(d_{i}, x_{-i}\right)>\alpha_{i}$.

It is clear that every weakly reciprocally upper semicontinuous game is transfer reciprocally upper semicontinuous. 
Reny's (1999) equilibrium existence theorem states that every compact, quasiconcave, better-reply secure game has a Nash equilibrium in pure strategies.

Theorem 1 (Reny,1999) If $G=\left(X_{i}, u_{i}\right)_{i \in I}$ is compact, quasiconcave, and better-reply secure, then it possesses a pure strategy Nash equilibrium.

Another approach to studying equilibrium existence in discontinuous games, based on the concept of diagonal transfer continuity, due to Baye, Tian, and Zhou (1993).

For $G=\left(X_{i}, u_{i}\right)_{i \in I}$, define the following aggregator functions:

$$
A_{G}: X \times X \rightarrow \mathbb{R} \text { by } A_{G}(d, x)=\sum_{i \in I} u_{i}\left(d_{i}, x_{-i}\right),
$$

where, as usual, the $-i$ subscript on $x$ stands for "all players except $i$,"

$$
A_{G}^{0}: X \rightarrow \mathbb{R} \text { by } A_{G}^{0}(x)=\sum_{i \in I} u_{i}(x)
$$

and

$$
F_{G}: X \times X \rightarrow \mathbb{R} \text { by } F_{G}(d, x)=A_{G}(d, x)-A_{G}^{0}(x) \text {. }
$$

A strategy profile $x \in X$ is a Nash equilibrium of $G$ iff $F_{G}(d, x) \leq 0$ for all $d \in X$.

Definition 5 A game $G=\left(X_{i}, u_{i}\right)_{i \in I}$ is diagonally transfer continuous if for every $x \in X \backslash E_{G}$, there exist some $d \in X$ and some neighborhood $\mathcal{N}_{X}(x)$ of $x$ in $X$ such that $F_{G}(d, z)>0$ for all $z \in \mathcal{N}_{X}(x)$.

It is worth noticing that $G$ is diagonally transfer continuous iff $F_{G}$ is 0 -transfer lower semicontinuous in $x$.

Every payoff secure game with an upper semicontinuous $A_{G}^{0}$ is diagonally transfer continuous. 
Lemma $1 \mathrm{If}$, in a game $G=\left(X_{i}, u_{i}\right)_{i \in I}$, each $u_{i}: X \rightarrow \mathbb{R}$ is transfer lower semicontinuous in $x_{-i}$ and the aggregator function $A_{G}^{0}: X \rightarrow \mathbb{R}$ is upper semicontinuous, then $G$ is diagonally transfer continuous.

For convenience, the proof of Lemma 1 is given in the Appendix.

Now we define the mixed extension $\Gamma(G)$ of a game $G=\left(X_{i}, u_{i}\right)_{i \in I}$. Denote by $\triangle\left(X_{i}\right)$ the set of Borel probability measures on $X_{i}$ and by $c a\left(X_{i}\right)$ the set of Borel signed measures with finite total variation on $X_{i}$. A basic open neighborhood of $\mu_{i} \in c a\left(X_{i}\right)$ in the weak topology on $c a\left(X_{i}\right)$ is a set of the form $\left\{\nu_{i} \in c a\left(X_{i}\right):\left|\int f_{j}\left(d \nu_{i}-d \mu_{i}\right)\right|<\varepsilon, j=1, \ldots, m\right\}$ for some continuous $f_{j}: X_{i} \rightarrow \mathbb{R}, j=1, \ldots, m$, and $\varepsilon>0$. The set $c a\left(X_{i}\right)$ is a Hausdorff topological vector space equipped with the weak topology. The topology induced on $\triangle\left(X_{i}\right)$ by the weak topology is compact. ${ }^{6}$ Let each of the Cartesian products $c a(X)=c a\left(X_{1}\right) \times \ldots \times c a\left(X_{n}\right)$ and $\triangle(X)=\triangle\left(X_{1}\right) \times \ldots \times \triangle\left(X_{n}\right)$ be equipped with the product topology. The set $c a(X)$ is a Hausdorff topological vector space in which the operations of addition and scalar multiplication are defined as follows: for $\mu=\left(\mu_{1}, \ldots, \mu_{n}\right) \in c a(X)$ and $\alpha \in \mathbb{R}$ the scalar multiplication of $\mu$ by $\alpha$ is the element $\alpha \mu$ given by $\alpha \mu=\left(\alpha \mu_{1}, \ldots, \alpha \mu_{n}\right)$. The addition of $\mu=\left(\mu_{1}, \ldots, \mu_{n}\right) \in c a(X)$ and $v=\left(\nu_{1}, \ldots, \nu_{n}\right) \in c a(X)$ gives $\mu+v=\left(\mu_{1}+\nu_{1}, \ldots, \mu_{n}+\nu_{n}\right)$.

The mixed extension of the game $G$ is the $n$-player normal form game $\Gamma(G)=\left(\triangle\left(X_{i}\right), U_{i}\right)_{i \in I}$, where $\triangle\left(X_{i}\right)$ is player $i$ 's strategy set and player $i$ 's payoff function $U_{i}: \triangle(X) \rightarrow \mathbb{R}$ is defined by

$$
U_{i}(\mu)=\int_{X_{1}} \int_{X_{2}} \ldots \int_{X_{n}} u_{i}\left(x_{1}, \ldots, x_{n}\right) d \mu_{1} \ldots d \mu_{n} .
$$

For the game $\Gamma(G)$, we also define the aggregator functions $A_{\Gamma(G)}$ :

\footnotetext{
${ }^{6}$ In order to make $\triangle\left(X_{i}\right)$ a subset of a linear space, we embed it in the space $c a\left(X_{i}\right)$ of signed measures with finite total variation on $X_{i}$. Sometimes it is possible to proceed without the embedding. See, for example, the proof of the compactness of the set of probability measures given by Glycopantis and Muir (2004).
} 
$\triangle(X) \times \triangle(X) \rightarrow \mathbb{R}, A_{\Gamma(G)}^{0}: \triangle(X) \rightarrow \mathbb{R}$, and $F_{\Gamma(G)}: \triangle(X) \times \triangle(X) \rightarrow \mathbb{R}$ (see the corresponding definitions for $G$ ).

Theorem 2 If the mixed extension $\Gamma(G)$ of a game $G=\left(X_{i}, u_{i}\right)_{i \in I}$ is diagonally transfer continuous, then $G$ possesses a mixed strategy Nash equilibrium.

Proof. The set $\triangle(X)$ is a compact, convex subset of $c a(X)$. Consider the aggregator function $F_{\Gamma(G)}(\sigma, \mu): \triangle(X) \times \triangle(X) \rightarrow \mathbb{R}$. Since $F_{\Gamma(G)}$ is linear in $\sigma$ and 0 -transfer lower semicontinuous in $\mu$, the mixed extension $\Gamma(G)$ of $G$ has a Nash equilibrium in pure strategies by the Ky Fan minimax inequality (see Lemma 4 in the Appendix).

Another proof of Theorem 2 can be obtained by using the fact that every diagonally transfer continuous mixed extension has the single deviation property (see, for some details, Reny, 2009, 2011; and Prokopovych, 2013).

Since the upper semicontinuity of $A_{G}^{0}$ implies the upper semicontinuity of $A_{\Gamma(G)}^{0}$, verifying whether a game has a mixed strategy Nash equilibrium usually means verifying the following two properties: (a) the upper semicontinuity of the sum of the payoff functions; and (b) the payoff security of its mixed extension. If these properties hold, the mixed extension of the game is not only better-reply secure but, by Lemma 1, diagonally transfer continuous. For example, this is the case for the all-pay auction games with homogeneous valuations (see Baye, Kovenock, and Vries, 1996; Monteiro and Page, 2007). It is also clear that every game whose payoff functions are continuous has a diagonally transfer continuous mixed extension since the mixed extension itself is a continuous game (see, e.g., Aliprantis, Glycopantis, and Puzzello, 2006).

The diagonal transfer continuity of a game does not imply that its mixed extension is diagonally transfer continuous. For example, Sion and Wolfe's (1957) zero-sum game is payoff secure (see Carmona, 2005) and its aggregator function $A_{G}^{0}$ is constant. Thus, the game is diagonally transfer continuous by 
Lemma 1. However, since the game has no mixed strategy Nash equilibria, its mixed extension is not diagonally transfer continuous.

\section{Uniform Diagonal Security}

An easily verifiable condition for the mixed extension of a game to be payoff secure is that of uniform payoff security, due to Monteiro and Page (2007).

Definition 6 A game $G=\left(X_{i}, u_{i}\right)_{i \in I}$ is uniformly payoff secure if for each $i \in I$, every $x_{i} \in X_{i}$ and every $\varepsilon>0$, there is $d_{i}\left(x_{i}, \varepsilon\right) \in X_{i}$ such that for every $x_{-i} \in X_{-i}, u_{i}\left(d_{i}\left(x_{i}, \varepsilon\right), w_{-i}\right) \geq u_{i}\left(x_{i}, x_{-i}\right)-\varepsilon$ for all $w_{-i}$ in some open neighborhood $\mathcal{N}_{X_{-i}}\left(x_{-i}\right)$ of $x_{-i}$ in $X_{-i}$.

We will omit the arguments $x_{i}$ and $\varepsilon$ of $d_{i}$ if there is no ambiguity. Another useful definition is the following: In $G=\left(X_{i}, u_{i}\right)_{i \in I}$, player $i$ 's payoff function $u_{i}: X_{i} \times X_{-i} \rightarrow \mathbb{R}$ is said to be uniformly transfer lower semicontinuous in $x_{-i}$ at $x_{i} \in X_{i}$ if for every $\varepsilon>0$, there is $d_{i} \in X_{i}$ such that, for every $x_{-i} \in X_{-i}$, there exists a neighborhood $\mathcal{N}_{X_{-i}}\left(x_{-i}\right)$ of $x_{-i}$ in $X_{-i}$ such that $u_{i}\left(d_{i}, w_{-i}\right)>u_{i}\left(x_{i}, x_{-i}\right)-\varepsilon$ for all $w_{-i} \in \mathcal{N}_{X_{-i}}\left(x_{-i}\right)$.

The mixed extension of a uniformly payoff secure, upper semicontinuoussum game is both better-reply secure and diagonally transfer continuous.

Corollary 1 If $G=\left(X_{i}, u_{i}\right)_{i \in I}$ is uniformly payoff secure and its aggregator function $A_{G}^{0}$ is upper semicontinuous, then the mixed extension $\Gamma(G)$ is diagonally transfer continuous, and, therefore, $G$ possesses a mixed strategy Nash equilibrium.

The notion of a uniformly payoff secure game can be extended to diagonally transfer continuous games.

Definition 7 A game $G=\left(X_{i}, u_{i}\right)_{i \in I}$ is uniformly diagonally secure if for every $d \in X$ and every $\varepsilon>0$, there is $\bar{d} \in X$ such that for every $x \in X$, 
$F_{G}(\bar{d}, w)>F_{G}(d, x)-\varepsilon$ for all $w$ in some open neighborhood $\mathcal{N}_{X}(x)$ of $x$ in $X$.

An upper semicontinuous-sum game $G$ is uniformly diagonally secure if it is uniformly payoff secure.

Lemma 2 If a game $G=\left(X_{i}, u_{i}\right)_{i \in I}$ is uniformly payoff secure and the aggregator function $A_{G}^{0}: X \rightarrow \mathbb{R}$ is upper semicontinuous, then $G$ is uniformly diagonally secure.

Proof. Fix $d \in X$ and $\varepsilon>0$. By the uniform payoff security of $G$, for each $i \in I$, there is a deviation strategy $\bar{d}_{i} \in X_{i}$ such that, for every $x_{-i} \in X_{-i}$, $u_{i}\left(\bar{d}_{i}, w_{-i}\right) \geq u_{i}\left(d_{i}, x_{-i}\right)-\frac{\varepsilon}{2 n}$ for all $w_{-i}$ in some open neighborhood $\mathcal{N}_{X_{-i}}\left(x_{-i}\right)$ of $x_{-i}$ in $X_{-i}$. Denote $\mathcal{N}_{X}^{1}(x)=\cap_{i \in I}\left\{X_{i} \times \mathcal{N}_{X_{-i}}\left(x_{-i}\right)\right\}$ for $x \in X$. Then $A_{G}(\bar{d}, w) \geq A_{G}(d, x)-\frac{\varepsilon}{2}$ for all $w \in \mathcal{N}_{X}^{1}(x)$. Since the function $-A_{G}^{0}$ is lower semicontinuous on $X$, for every $x \in X$ there exists a neighborhood $\mathcal{N}_{X}^{2}(x)$ such that $-A_{G}^{0}(w)>-A_{G}^{0}(x)-\frac{\varepsilon}{2}$ for all $w \in \mathcal{N}_{X}^{2}(x)$. Then for every $x \in X$, $F_{G}(\bar{d}, w)>F_{G}(d, x)-\varepsilon$ for all $w \in \mathcal{N}_{X}^{1}(x) \cap \mathcal{N}_{X}^{2}(x)$.

Theorem 3 If a game $G=\left(X_{i}, u_{i}\right)_{i \in I}$ is uniformly diagonally secure, then its mixed extension $\Gamma(G)$ is diagonally transfer continuous, and, therefore, $G$ possesses a mixed strategy Nash equilibrium.

The proof of Theorem 3 follows the lines of the proof of Theorem 1 of Monteiro and Page (2007) and is given in the Appendix.

The concept of uniform diagonal security might be of help in studying equilibirum existence in games whose aggregator function $A_{G}^{0}$ is not upper semicontinuous.

Example 1 Consider a slight modification of the rent-seeking game due to Tullock (1980). Two players simultaneously bid for a political favor commonly known worth $V$ dollars. Their bids, denoted by $x_{1}$ and $x_{2}$, influence the probability of receiving the favor. Player $i$ 's strategy set is the segment 
$[0, V]$. Let $\pi_{i}\left(x_{1}, x_{2}\right)$ denote the probability that player $i$ wins. The function $\pi_{i}$, called player $i$ 's contest success function, is specified as follows:

$$
\pi_{i}\left(x_{i}, x_{-i}\right)=\left\{\begin{array}{c}
\frac{1}{4} \text { if } x_{1}=x_{2}=0 \\
\frac{1}{2} \text { if } x_{i}>x_{-i}=0 \\
\frac{x_{i}^{r}}{x_{i}^{r}+x_{-i}^{r}} \text { otherwise }
\end{array}\right.
$$

where $r>0$. Player $i$ 's payoff function $u_{i}$ is

$$
u_{i}\left(x_{i}, x_{-i}\right)=\pi_{i}\left(x_{i}, x_{-i}\right) V-x_{i}
$$

The only difference of the model from the Tullock rent-seeking game is the assumption that if the lowest bid submitted is equal to zero (or, in other words, at least one player exerts no effort at all), the favor may be granted to a third party with probability one-half. Consequently, the aggregator function $A_{G}^{0}$ is not upper semicontinuous.

Let, for specificity, $V=2$ and $r=3$. In this case, the game has no pure strategy Nash equilibria (see, for a related discussion, Baye, Kovenock, and de Vries, 1994). For example, one can check that the only candidate point for being an interior solution is $(1.5,1.5)$, a strategy profile where both players get negative expected payoffs. However, each of them can avoid getting a negative payoff by bidding zero.

To verify that the game is not better-reply secure, consider the sequence $\left\{x^{k}\right\}$ with $x^{k}=\left(\frac{1}{k}, \frac{1}{k}\right)$ for $k=1,2, \ldots$ Then the corresponding sequence of payoff vectors $\left\{\left(u_{1}\left(x^{k}\right), u_{2}\left(x^{k}\right)\right)\right\}$ converges to $(1,1)$. It is clear that no player can secure a payoff strictly above 1 at $(0,0)$.

On the other hand, the game possesses mixed strategy equilibria since it is uniformly diagonally secure. To verify this, for $d \in X$ and $\varepsilon \in(0,1)$, define 
$\bar{d}=\left(\bar{d}_{1}, \bar{d}_{2}\right)$ as follows:

$$
\bar{d}_{i}=\left\{\begin{array}{l}
d_{i} \text { if } d_{i}>0, \\
\frac{\varepsilon}{4} \text { if } d_{i}=0,
\end{array} \quad \text { for } i=1,2\right.
$$

It is a little tedious but not difficult to show that, for every $x \in X, F_{G}(\bar{d}, w)>$ $F_{G}(d, x)-\varepsilon$ for all $w$ in some open neighborhood $\mathcal{N}_{X}(x) .{ }^{7}$

\section{Weak Uniform Payoff Security}

The Sion-Wolfe (1957) game is a better-reply secure game that has no Nash equilibria. Its mixed extension is upper semicontinuous-sum, but is not payoff secure (see Carmona, 2005, Example 3). From now on, we study equilibrium existence in games whose mixed extension is payoff secure. Example 2 shows that there are better-reply secure games with a payoff secure mixed extension which have no Nash equilibria.

The following definition extends Simon's (1987, p. 577) concept of weak domination on average to our setting.

Definition 8 In $G=\left(X_{i}, u_{i}\right)_{i \in I}$, player $i$ 's payoff function $u_{i}$ is weakly uniformly transfer lower semicontinuous in the other players' strategies at $x_{i} \in X_{i}$ if for every $\mu_{-i} \in \triangle\left(X_{-i}\right)$ and every $\varepsilon>0$, there are a strategy $\sigma_{i}\left(x_{i}, \mu_{-i}, \varepsilon\right) \in \triangle\left(X_{i}\right)$ and a Borel set $Q_{-i} \subset X_{-i}$ with $\mu_{-i}\left(Q_{-i}\right)>1-\varepsilon$ such that for every $x_{-i} \in Q_{-i}, U_{i}\left(\sigma_{i}\left(x_{i}, \mu_{-i}, \varepsilon\right), w_{-i}\right)>u_{i}\left(x_{i}, x_{-i}\right)-\varepsilon$ for all $w_{-i}$ in some open neighborhood $\mathcal{N}_{X_{-i}}\left(x_{-i}\right)$ of $x_{-i}$ in $X_{-i}$. The game $G$ is weakly uniformly payoff secure if each $u_{i}$ is weakly uniformly transfer lower semicontinuous in the other players' strategies at every $x_{i} \in X_{i}$.

\footnotetext{
${ }^{7}$ If needed, a detailed explanation of this example can be found in the appendix of our working paper titled "On Uniform Conditions for the Existence of Mixed Strategy Equilibria" at SSRN: http://ssrn.com/abstract=2023500 or http://dx.doi.org/10.2139/ssrn.2023500.
} 
There is some notational abuse in denoting $\int_{X_{i}} u_{i}\left(x_{i}, w_{-i}\right) d \sigma_{i}\left(x_{i}, \mu_{-i}, \varepsilon\right)$ by $U_{i}\left(\sigma_{i}\left(x_{i}, \mu_{-i}, \varepsilon\right), w_{-i}\right)$, but no ambiguity ensues. The proof of the next statement follows the lines of the proof of Proposition 3 of Simon (1987) and is relegated to the Appendix.

Lemma 3 If a game $G=\left(X_{i}, u_{i}\right)_{i \in I}$ is weakly uniformly payoff secure, then its mixed extension $\Gamma(G)$ is payoff secure.

The game studied in Example 2 is not only better-reply secure but also has a payoff secure mixed extension. However it does not possess mixed strategy Nash equilibria.

Example 2 Let $I=\{1,2\}, X=[0,1] \times[0,1]$, and the payoff functions are defined by

$$
\begin{aligned}
& u_{1}\left(x_{1}, x_{2}\right)=\left\{\begin{array}{l}
0 \text { if } x_{2}=x_{1}+\frac{1}{2}, x_{2}=x_{1}, \text { or }\left(x_{1}, x_{2}\right)=(0,1), \\
x_{2}-x_{1}-1 \text { if } 0 \leq x_{1}<x_{2}<x_{1}+\frac{1}{2}, \\
1 \text { otherwise },
\end{array}\right. \\
& u_{2}\left(x_{2}, x_{1}\right)=\left\{\begin{array}{l}
0 \text { if } x_{2}=x_{1}+\frac{1}{2}, x_{2}=x_{1}, \text { or }\left(x_{1}, x_{2}\right) \in\left[0, \frac{1}{2}\right) \times\{1\}, \\
1 \text { if } 0 \leq x_{1}<x_{2}<x_{1}+\frac{1}{2}, \\
-1 \text { otherwise }
\end{array}\right.
\end{aligned}
$$

Let us show that the game has no mixed strategy Nash equilibria. It is clear that there is no Nash equilibrium $\left(\widehat{\mu}_{1}, \widehat{\mu}_{2}\right)$ with $\widehat{\mu}_{2}(\{1\})=1$.

Assume, by way of contradiction, that $\left(\widehat{\mu}_{1}, \widehat{\mu}_{2}\right)$ is a mixed strategy Nash equilibrium of the game.

If $\widehat{\mu}_{2}(\{1\})=0$, then the set $D=\left\{\left(x_{1}, x_{2}\right) \in X: \min \left\{0, x_{2}-\frac{1}{2}\right\} \leq x_{1} \leq\right.$ $\left.x_{2}\right\}$ must be a null set with respect to the product measure $\widehat{\mu}=\widehat{\mu}_{1} \times \widehat{\mu}_{2}$, otherwise, by choosing strategy 1 with probability 1 , player 1 can increase her payoff. Then, in the equilibrium, the payoff to player 2 is equal to -1 . However, player 2 can guarantee herself at least 0 by choosing strategy 1 with probability 1 , a contradiction. Therefore, $\widehat{\mu}_{2}(\{1\})>0$. Then it must also be the case that $\widehat{\mu}_{1}\left(\left(\frac{1}{2}, 1\right)\right)=0$. 
The set $D_{1}=\left\{\left(x_{1}, x_{2}\right) \in X: 0 \leq x_{2} \leq x_{1}, 0 \leq x_{1}<\frac{1}{2}\right\}$ must be a null set with respect to the product measure $\widehat{\mu}$, otherwise player 2 can increase her payoff by shifting some weight from $\left[0, \frac{1}{2}\right)$ closer to $\frac{1}{2}$. The set $D_{2}=\left\{\left(x_{1}, x_{2}\right) \in X: x_{1}<x_{2} \leq x_{1}+\frac{1}{2}, 0<x_{1}<\frac{1}{2}\right\}$ is also a null set, otherwise player 1 could benefit from shifting some weight from $\left(0, \frac{1}{2}\right)$ closer to 0 (recall that, on $D_{2}, u_{1}\left(\cdot, x_{2}\right)$ is a strictly decreasing function for any $\left.x_{2} \in(0,1)\right)$. Furthermore, the set $D_{3}=\left\{\left(x_{1}, x_{2}\right) \in X: x_{1}+\frac{1}{2}<x_{2}<1\right.$, $\left.x_{1} \neq 0\right\}$ is a null set, otherwise player 2 can increase her payoff by shifting some weight from $\left(\frac{1}{2}, 1\right)$ closer to $\frac{1}{2}$.

If $\widehat{\mu}_{1}\left(\left(0, \frac{1}{2}\right)\right)>0$, then the fact that $D_{1} \cup D_{2} \cup D_{3}$ is a null set implies that $\widehat{\mu}_{2}([0,1))=0$, which is impossible since there is no Nash equilibrium with $\widehat{\mu}_{2}(\{1\})=1$. Thus, $\widehat{\mu}_{1}\left(\{0\} \cup\left\{\frac{1}{2}\right\} \cup\{1\}\right)=1$.

If $\widehat{\mu}_{2}\left(\left[0, \frac{1}{2}\right]\right)>0$, then $\widehat{\mu}_{1}(\{0\})=0$, otherwise player 1 can increase her payoff by shifting the mass $\widehat{\mu}_{1}(\{0\})$ to strategy 1 (recall that $u_{1}(0,1)=0$ ). Further, player 2 can increase her payoff by shifting the mass $\widehat{\mu}_{2}\left(\left[0, \frac{1}{2}\right]\right)$ to strategy 1 if $\widehat{\mu}_{1}(\{1\})>0$, and, for example, to $\frac{2}{3}$ if $\widehat{\mu}_{1}(\{1\})=0$, a contradiction.

If $\widehat{\mu}_{2}\left(\left(\frac{1}{2}, 1\right)\right)>0$, then $\widehat{\mu}_{1}\left(\left\{\frac{1}{2}\right\}\right)=0\left(\right.$ since $\left.\widehat{\mu}_{2}\left(\left(\frac{1}{2}, 1\right]\right)=1\right)$, and again player 2 can get a nonnegative payoff by playing 1 with probability 1 . Thus, $\widehat{\mu}_{2}(\{1\})=1$, a contradiction.

We will now show, for completeness, that $u_{1}$ is weakly uniformly transfer lower semicontinuous in player 2's strategies at every $x_{1} \in X_{1}$. Denote by $\delta_{x_{1}}^{D}$ the Dirac measure concentrated at $x_{1}$. One can see that the payoff function $u_{1}$ is uniformly transfer lower semicontinuous in player 2's strategies at any $x_{1} \in\{0\} \cup\left[\frac{1}{2}, 1\right]$, with $\sigma_{1}\left(x_{1}, \mu_{2}, \varepsilon\right)=\delta_{1}^{D}$ for every $\mu_{2} \in \triangle\left(X_{2}\right)$, and every $\varepsilon>0$. Fix some $x_{1} \in\left(0, \frac{1}{2}\right), \mu_{2} \in \triangle\left(X_{2}\right)$, and $\varepsilon>0$. It is not difficult to see that there is $\varepsilon^{\prime} \in(0, \varepsilon)$ such that both $x_{1}-\varepsilon^{\prime}$ and $x_{1}+\varepsilon^{\prime}$ lie in the interval $\left(0, \frac{1}{2}\right), \mu_{2}\left(Q_{2}\right)>1-\varepsilon$ where $Q_{2}=X_{2} \backslash\left(\left[x_{1}-\varepsilon^{\prime}, x_{1}\right) \cup\left(x_{1}, x_{1}+\varepsilon^{\prime}\right] \cup\left[x_{1}+\frac{1}{2}-\right.\right.$ $\left.\left.\varepsilon^{\prime}, x_{1}+\frac{1}{2}\right) \cup\left(x_{1}+\frac{1}{2}, x_{1}+\frac{1}{2}+\varepsilon^{\prime}\right]\right)$, and $U_{1}\left(\frac{1}{2} \delta_{x_{1}-\varepsilon^{\prime}}^{D}+\frac{1}{2} \delta_{x_{1}+\varepsilon^{\prime}}^{D}, w_{2}\right)>u_{1}\left(x_{1}, x_{2}\right)-\varepsilon$ for every $x_{2} \in Q_{2}$ and all $w_{2}$ in some open neighborhood $\mathcal{N}_{X_{2}}\left(x_{2}\right)$ of $x_{2}$ in 
$X_{2}$. A similar reasoning can be used to show that $u_{2}$ is weakly uniformly transfer lower semicontinuous in player 1's strategies at every $x_{2} \in X_{2}$.

\section{Equilibria of Reciprocally Upper Semicon- tinuous Games with Payoff Secure Mixed Extensions}

Verifying whether a game is transfer reciprocally upper semicontinuous is often considerably easier than doing that for its mixed extension. In this section, we study several classes of better-reply secure games with payoff secure mixed extensions that also have transfer reciprocally upper semicontinuous mixed extensions.

Some reciprocally upper semicontinuous games can be transformed into upper semicontinuous-sum games with the aid of positive affine transformations. If this is the case, the game has a reciprocally upper semicontinuous mixed extension. Example 3 demonstrates that a conventional probabilistic spatial voting model possesses this property.

Then we study equilibrium existence in reciprocally upper semicontinuous games of two players on the unit square that have a payoff secure mixed extension . Additional conditions are made on the set of discontinuities of the payoff functions. In particular, it is assumed that the discontinuities lie on one or two strictly monotone, continuous curves.

We now introduce some notation. Recall that a function $\phi: \mathbb{R} \rightarrow \mathbb{R}$ is a positive affine transformation if it can be written in the form: $\phi(x)=a x+b$ where $a>0$ and $b$ is any real number. Given a game $G=\left(X_{i}, u_{i}\right)_{i \in I}$ and an $n$-tuple of positive affine transformations $\left(\phi_{1}, \ldots, \phi_{n}\right)$, we denote by $\Phi(G)$ the game $\left(X_{i}, \phi_{i}\left(u_{i}\right)\right)_{i \in I}$. The following three facts are straightforward.

(i) Let $G=\left(X_{i}, u_{i}\right)_{i \in I}$, and let $\left(\phi_{1}, \ldots, \phi_{n}\right)$ be an $n$-tuple of positive affine transformations. Then $E_{\Gamma(G)}=E_{\Gamma(\Phi(G))}$. 
(ii) Let $\left(\phi_{1}, \ldots, \phi_{n}\right)$ be an $n$-tuple of positive affine transformations. Then $G=\left(X_{i}, u_{i}\right)_{i \in N}$ is weakly uniformly payoff secure iff the game $\Phi(G)$ is weakly uniformly payoff secure.

(iii) Let $\left(\phi_{1}, \ldots, \phi_{n}\right)$ be an $n$-tuple of positive affine transformations. Then $G=\left(X_{i}, u_{i}\right)_{i \in N}$ is reciprocally upper semicontinuous iff the game $\Phi(G)$ is reciprocally upper semicontinuous.

Theorem 4 If $G=\left(X_{i}, u_{i}\right)_{i \in I}$ is weakly uniformly payoff secure, and there is an $n$-tuple of positive affine transformations $\left(\phi_{1}, \ldots, \phi_{n}\right)$ such that the game $\Phi(G)$ is upper semicontinuous-sum, then $G$ possesses a mixed strategy Nash equilibrium.

Proof. Since $G$ is weakly uniformly payoff secure, the game $\Phi(G)$ is also weakly uniformly payoff secure by fact (ii). Thus, $\Gamma(\Phi(G))$ is payoff secure by Lemma 3.

The fact that $\Phi(G)$ is upper semicontinuous-sum implies that $\Gamma(\Phi(G))$ is upper semicontinuous-sum. Since $\Gamma(\Phi(G))$ is the same game as $\Phi(\Gamma(G))$, the mixed extension $\Gamma(G)$ is reciprocally upper semicontinuous by fact (iii). By Theorem 1, $\Gamma(G)$ possesses a pure strategy Nash equilibrium.

Example 3 Consider the following spatial voting model (see Ball, 1999, Example 1). Two candidates are competing in an election for public office. The electorate is distributed uniformly along the ideological spectrum $[0,1]$. During the electoral campaign, each candidate $i$ announces, simultaneously with the other candidate, a platform, denoted by $x_{i}$. The probability $P_{i}\left(x_{i}, x_{-i}\right)$ that candidate $i$ wins the election is defined as follows:

$$
P_{i}\left(x_{i}, x_{-i}\right)=\left\{\begin{array}{l}
\frac{x_{i}+x_{-i}}{2} \text { for } 0 \leq x_{i}<x_{-i} \leq 1, \\
\frac{1}{2} \text { for } 0 \leq x_{i}=x_{-i} \leq 1 \\
1-\frac{x_{i}+x_{-i}}{2} \text { for } 0 \leq x_{-i}<x_{i} \leq 1
\end{array}\right.
$$

Candidates 1 and 2's policy preferences on $[0,1]$ are represented by $h_{1}(z)=$ $-\frac{1}{2}(z-1)^{2}$ and $h_{2}(z)=-\frac{1}{2} z^{2}$. The candidates are assumed to be officemotivated. Let the candidates' office motivation parameters be $k_{1}=.05$ and 
$k_{2}=3$, respectively. Candidate $i$ 's payoff function is

$$
u_{i}\left(x_{i}, x_{-i}\right)=P_{i}\left(x_{i}, x_{-i}\right)\left(h_{i}\left(x_{i}\right)+k_{i}\right)+\left(1-P_{i}\left(x_{i}, x_{-i}\right)\right) h_{i}\left(x_{-i}\right) .
$$

The game is not upper semicontinuous-sum and has no pure strategy Nash equilibria.

Now we consider the game $\Phi(G)=\left(X_{i}, \phi_{i}\left(u_{i}\right)\right)_{i \in\{1,2\}}$ where $\phi_{1}$ and $\phi_{2}$ are defined by $\phi_{1}(t)=\frac{k_{2}}{k_{1}} t$ and $\phi_{2}(t)=t$ for $t \in \mathbb{R}$, respectively. It is not difficult to see that $A_{\Phi(G)}^{0}$ is a continuous function on $X$. Since $G$ is uniformly payoff secure, $G$ possesses a mixed strategy Nash equilibrium by Theorem 4 .

It is useful to notice that the voting game is an example of a game with a better-reply secure mixed extension that is not upper semicontinuous-sum.

In the next two theorems, we study the existence of mixed strategy Nash equilibria in some basic better-reply secure games of two players. To start with, we consider the case when all discontinuities of the payoff functions lie on a strictly monotonic, continuous curve. Denote $S^{1}=\left\{\left(x_{1}, x_{2}\right) \in\right.$ $\left.[0,1] \times[0,1]: x_{2}>x_{1}\right\}, S^{2}=\left\{\left(x_{1}, x_{2}\right) \in[0,1] \times[0,1]: x_{1}>x_{2}\right\}$, and $S=\left\{\left(x_{1}, x_{2}\right):[0,1] \times[0,1]: x_{1}=x_{2}\right\}$.

Theorem 5 Let $G=\left(X_{i}, u_{i}\right)_{i \in\{1,2\}}$ be a two-player game on the unit square $X_{1} \times X_{2}=[0,1] \times[0,1]$. Suppose that

(i) $G$ is uniformly payoff secure and reciprocally upper semicontinuous;

(ii) there are continuous functions $f_{i}^{j}: \operatorname{cl}^{j} \rightarrow \mathbb{R}, i=1,2, j=1,2$, such that $u_{i}(x)=f_{i}^{j}(x)$ for all $x \in S^{j}$ and all $i, j \in\{1,2\}$;

(iii) $u_{1}(0,0) \leq f_{1}^{2}(0,0)$ and $u_{1}(1,1) \leq f_{1}^{1}(1,1)$;

(iv) the restriction of each $u_{i}$ to $S, u_{i \mid S}$, is a continuous function from $S$ to $\mathbb{R}$.

Then $G$ has a better-reply secure mixed extension, and, therefore, it has a mixed strategy Nash equilibrium.

Condition (ii) is stronger than the assumption that all points of discontinuity of the payoff functions lie on $S$. In particular, (ii) implies that 
$\lim _{k \rightarrow \infty} u_{i}\left(x^{k}\right)=f_{i}^{j}(x)$ for every sequence of points $\left\{x^{k}\right\}, x^{k} \in S^{j}$, that converges to $x \in S$; that is, the limit value does not depend on the choice of a sequence in $S^{j}$. Condition (iii) may be replaced, if needed, with a number of other conditions, such as: (iii') for every $\varepsilon>0$ and each $z \in\{0,1\}$, there exists a deviation strategy $d_{1}(z, \varepsilon)$ satisfying the uniform payoff security condition for player 1's strategy $z$ that is different from $z$; or (iii") $u_{2}(0,0) \leq f_{2}^{1}(0,0)$ and $u_{2}(1,1) \leq f_{2}^{2}(1,1)$.

Theorem 5 can be extended to a class of games where the discontinuities of the payoff functions might lie on two strictly monotone, disjoint curves. In particular, Theorem 6 covers the games where one of the curves is strictly increasing and the other is strictly decreasing. ${ }^{8}$ We will show it for a representative game from this class. For every $h: \mathbb{R}^{1} \rightarrow \mathbb{R}^{1}$, denote $X_{h}^{1}=\left\{\left(x_{1}, x_{2}\right): h\left(x_{1}\right)<x_{2}\right\}, X_{h}^{2}=\left\{\left(x_{1}, x_{2}\right): h\left(x_{1}\right)>x_{2}\right\}$, and $X_{h}=\left\{\left(x_{1}, x_{2}\right): h\left(x_{1}\right)=x_{2}\right\}$.

Theorem 6 Let $G=\left(X_{i}, u_{i}\right)_{i \in\{1,2\}}$ be a two-player game on the unit square $X_{1} \times X_{2}=[0,1] \times[0,1]$, and let $h_{1}: \mathbb{R}^{1} \rightarrow \mathbb{R}^{1}$ be defined by $h_{1}\left(x_{1}\right)=\frac{1}{4} x_{1}+\frac{3}{4}$ and $h_{2}: \mathbb{R}^{1} \rightarrow \mathbb{R}^{1}$ by $h_{2}\left(x_{1}\right)=-\frac{1}{4} x_{1}+\frac{1}{4}$. Suppose that

(i) $G$ is uniformly payoff secure and reciprocally upper semicontinuous;

(ii) for each $i \in\{1,2\}$, there are continuous functions $f_{i}^{1}: \operatorname{cl} X_{h_{1}}^{1} \rightarrow \mathbb{R}, f_{i}^{2}$ : $\operatorname{cl} X_{h_{1}}^{2} \cap \operatorname{cl} X_{h_{2}}^{1} \rightarrow \mathbb{R}$, and $f_{i}^{3}: \operatorname{cl} X_{h_{2}}^{2} \rightarrow \mathbb{R}$ such that $u_{i}(x)=f_{i}^{1}(x)$ for every $x \in$ $X_{h_{1}}^{1}, u_{i}(x)=f_{i}^{2}(x)$ for every $x \in X_{h_{1}}^{2} \cap X_{h_{2}}^{1}$, and $u_{i}(x)=f_{i}^{3}(x)$ for every $x \in X_{h_{2}}^{2}$;

(iii) $u_{1}(1,1) \leq f_{1}^{1}(1,1)$ and $u_{1}(1,0) \leq f_{1}^{3}(1,0)$;

(iv) for each $i \in\{1,2\}$ and each $l \in\{1,2\}$, the restriction of $u_{i}$ to $X_{h_{l}}$, $u_{i \mid X_{h_{l}}}$, is a continuous function from $X_{h_{l}}$ to $\mathbb{R}$.

Then $G$ has a better-reply secure mixed extension, and, therefore, it has a mixed strategy Nash equilibrium.

Condition (iii) of Theorem 6 may be replaced with a number of other

\footnotetext{
${ }^{8}$ Equilibrium existence conditions similar to those presented in Theorem 6 can also be provided for Sion-Wolfe-type games.
} 
geometric conditions, if need appears.

Example 4 Consider the following deterministic spatial voting model. Two candidates are competing in an election for public office. The electorate is distributed uniformly along the ideological spectrum $[0,1]$. The candidate with the most votes wins. Each voter casts his vote for the candidate that is closest to her ideological position. During the electoral campaign, each candidate $i$ announces, simultaneously with the other candidate, a platform $x_{i} \in[0,1]$ to which he commits if elected. The probability that candidate $i$ wins the election is

$$
P_{i}\left(x_{i}, x_{-i}\right)=\left\{\begin{array}{l}
1, \text { if }\left(x_{i}+x_{-i}-1\right)\left(x_{-i}-x_{i}\right)>0 \\
\frac{1}{2} \text { if } x_{i}+x_{-i}=1 \text { or } x_{i}=x_{-i} \\
0, \text { if }\left(x_{i}+x_{-i}-1\right)\left(x_{-i}-x_{i}\right)<0
\end{array}\right.
$$

The inequality $\left(x_{i}+x_{-i}-1\right)\left(x_{-i}-x_{i}\right)>0$ represents the fact that candidate $i$ gets most of the votes. For example, if $x_{-i}>x_{i}$ and $\frac{x_{i}+x_{-i}}{2}>\frac{1}{2}$, then $\left(x_{i}+x_{-i}-1\right)\left(x_{-i}-x_{i}\right)>0$. The candidates' loss functions on $[0,1]$ are represented by $h_{1}(z)=-\frac{1}{2}(z-1)^{2}$ and $h_{2}(z)=-\frac{1}{2} z^{2}$, and the candidates are assumed to be office-motivated. Let the office motivation parameters be $k_{1}=.05$ and $k_{2}=3$, respectively. Candidate $i$ 's payoff function is

$$
u_{i}\left(x_{i}, x_{-i}\right)=h_{i}\left(x_{i}\right)+P_{i}\left(x_{i}, x_{-i}\right) k_{i} .
$$

Thus, each candidate cares only about winning, and taking an ideological position different from her ideal point is costly. It is not difficult to see that the game has no pure strategy Nash equilibria. Both Theorem 4 and Theorem 6 can be used to prove the existence of mixed strategy Nash equilibria in this game, notwithstanding the fact that the discontinuities of the payoff functions lie on both of the diagonals of the unit square.

We now show how to apply Theorem 6 to this game. First notice that every pure strategy $z \in\left[0, \frac{3}{5}\right)$ of player 1 is strictly dominated by strategy 1 . 
As a result, to show that the game under study has mixed strategy equilibria, it is enough to investigate whether its restriction $G^{r}$ to $\left[\frac{3}{5}, 1\right] \times[0,1]$ has mixed strategy equilibria, where the game $G^{r}=\left(X_{i}^{r}, u_{i}^{r}\right)_{i \in\{1,2\}}$ is defined as follows: $X_{1}^{r}=\left[\frac{3}{5}, 1\right], X_{2}^{r}=X_{2}=[0,1], u_{i}^{r}(x)=u_{i}(x)$ for every $x \in X_{1}^{r} \times X_{2}^{r}$ and each $i$.

One can see that $G^{r}$ is uniformly payoff secure. For example, given $\varepsilon>0$, if $x_{1}=\frac{3}{5}$, then for every $x_{2} \in[0,1]$ there exists an open neighborhood $\mathcal{N}_{X_{2}}\left(x_{2}\right)$ such that $u_{1}\left(1, w_{2}\right)>u_{1}\left(\frac{3}{5}, x_{2}\right)-\varepsilon$ for all $w_{2} \in \mathcal{N}_{X_{2}}\left(x_{2}\right)$. If $x_{1} \in\left(\frac{3}{5}, 1\right]$, then there is $\delta \in\left(0, x_{1}-\frac{3}{5}\right)$ such that for every $x_{2} \in[0,1], u_{1}\left(x_{1}-\delta, w_{2}\right)>$ $u_{1}\left(x_{1}, x_{2}\right)-\varepsilon$ for all $w_{2}$ in some neighborhood $\mathcal{N}_{X_{2}}\left(x_{2}\right)$ of $x_{2}$. Clearly, the game is reciprocally upper semicontinuous. The existence of a mixed strategy Nash equilibrium in $G^{r}$ follows from Theorem 6.

\section{Conclusions}

We use aggregator functions in conjunction with the Ky Fan minimax inequality to study the existence of a mixed strategy Nash equilibrium in diagonally transfer continuous games. Similar to the approach based on the concept of better-reply security, the aggregation-based approach is applicable to the upper semicontinuous-sum games whose mixed extension is payoff secure. However, if the sum of a game's payoff functions of a game is not upper semicontinuous, showing that its mixed extension is either better-reply secure or diagonally transfer continuous often constitutes an intractable problem. To alleviate it, the concept of uniform payoff security is extended to diagonally transfer continuous games by introducing uniform diagonal security. We show that every uniformly diagonally secure game possesses a mixed strategy Nash equilibrium, and, with the aid of an example, that uniformly diagonally secure games need not be better-reply secure.

After introducing a generalization of uniform payoff security, called weak uniform payoff security, we provide an example of a better-reply secure game 
with a payoff secure mixed extension that has no mixed strategy Nash equilibria. Then we study the existence of a mixed strategy Nash equilibrium in games that are reciprocally upper semicontinuous and uniformly payoff secure. We propose two sets of easily verifiable, geometric conditions for a better-reply secure game of two players to possess a better-reply secure mixed extension.

\section{Appendix}

\section{Proof of Lemma 1}

We shall first show that $A_{G}(d, x)$ is transfer lower semicontinuous in $x$. Let $(d, x) \in X \times X$ and $\lambda \in \mathbb{R}$ be such that $A_{G}(d, x)>\lambda$. Then there are $\lambda_{1}, \ldots, \lambda_{n} \in \mathbb{R}$ such that $\lambda=\lambda_{1}+\ldots+\lambda_{n}$ and $u_{i}\left(d_{i}, x_{-i}\right)>\lambda_{i}$ for all $i \in I$. Since each $u_{i}$ is transfer lower semicontinuous in $x_{-i}$, there exist $\bar{d}_{i} \in X_{i}$ and an open neighborhood $\mathcal{N}_{X_{-i}}\left(x_{-i}\right)$ of $x_{-i}$ in $X_{-i}$ such that $u_{i}\left(\bar{d}_{i}, z_{-i}\right)>\lambda_{i}$ for

all $z_{-i} \in \mathcal{N}_{X_{-i}}\left(x_{-i}\right)$. Consequently, $A_{G}(\bar{d}, z)>\lambda$ for every $z \in \cap_{i \in I}\left\{X_{i} \times\right.$ $\left.\mathcal{N}_{X_{-i}}\left(x_{-i}\right)\right\}$.

Since $A_{G}^{0}$ is upper semicontinuous on $X$, the transfer lower semicontinuity of $A_{G}$ in $x$ implies the transfer lower semicontinuity of $F_{G}$ in $x$. In particular, $F_{G}$ is 0-transfer lower semicontinuous in $x$.

\section{The Ky Fan Minimax Inequality}

In Theorem 2, the Ky Fan minimax inequality is used in the following, slightly generalized form. (see, e.g., Tian, 1992; Ding and Park, 2002; Lan and Wu, 2002, for more general results).

Lemma 4 Let $X$ be a compact convex set in a Hausdorff topological vector space, and let $f: X \times X \rightarrow \mathbb{R}$ satisfy:

(i) $f(x, x) \leq 0$ for each $x \in X$; 
(i) $f(\cdot, y)$ is quasiconcave for each $y \in X$,

(ii) $f$ is 0-transfer lower semicontinuous in $y$.

Then there exists $\bar{y} \in X$ such that $f(x, \bar{y}) \leq 0$ for all $x \in X$.

Lemma 4 can be shown in a number of ways. Its conventional proofs are based either on the KKM lemma or on Browder's fixed point theorem, which are two equivalent results (see, for an in-depth discussion, Yannelis, 1991). Let us give an outline of the proof using Browder's fixed point theorem. It proceeds by assuming, to the contrary, that, for each $y \in X$, there exists $x \in X$ such that $f(x, y)>0$. Then the correspondence $M: X \rightarrow X$ defined by $M(y)=\{x \in X: f(x, y)>0\}$ has nonempty values. The quasiconcavity of $f$ in $x$ implies that $M$ has convex values. Since $f$ is 0 transfer lower semicontinuous in $y, M$ has a multivalued selection with open lower sections (see, e.g., Prokopovych, 2011), denoted by $M_{0}: X \rightarrow X$. Then, by Lemma 5.1 of Yannelis and Prabhakar (1983), the convex-valued correspondence $\bar{M}_{0}: X \rightarrow X$ defined by $\bar{M}_{0}(x)=\operatorname{co} M_{0}(x)$ also has open lower sections. Therefore, by Browder's fixed point theorem, the selection has a fixed point, which contradicts (i).

\section{Proof of Theorem 3}

Suppose $\mu=\left(\mu_{1}, \ldots, \mu_{n}\right) \in \triangle(X) \backslash E_{\Gamma(G)}$. Then there exists $\sigma \in \triangle(X)$ such that $F_{\Gamma(G)}(\sigma, \mu)>0$. Since $\sigma$ is a vector of probability measures, there exists $d=\left(d_{1}, \ldots, d_{n}\right) \in X$ such that $F_{\Gamma(G)}\left(\delta_{d}^{D}, \mu\right)>0$, where $\delta_{d}^{D}=\left(\delta_{d_{1}}^{D}, \ldots, \delta_{d_{n}}^{D}\right)$ is the vector of Dirac measures concentrated at $d_{1}, \ldots, d_{n}$, respectively. With some abuse of notation, we will write $F_{\Gamma(G)}(d, \mu)$ in place of $F_{\Gamma(G)}\left(\delta_{d}^{D}, \mu\right)$. Put $\varepsilon^{*}=F_{\Gamma(G)}(d, \mu)$ and denote $\liminf _{w \rightarrow x} F_{G}(d, w)$ by $\underline{F}_{G}(d, x)$. Since $G$ is uniformly diagonally secure, there is $\bar{d} \in X$ such that $\underline{F}_{G}(\bar{d}, x)>F_{G}(d, x)-\frac{\varepsilon^{*}}{2}$ for all $x \in X$. Therefore $\underline{F}_{\Gamma(G)}(\bar{d}, \mu)>F_{\Gamma(G)}(d, \mu)-\frac{\varepsilon^{*}}{2}$, where $\underline{F}_{\Gamma(G)}(\bar{d}, \mu)=$ $\int_{X} \underline{F}_{G}(\bar{d}, x) d \mu$.

The lower semicontinuity of $\underline{F}_{G}$ in $x$ implies that $\underline{F}_{\Gamma(G)}(\bar{d}, \cdot): \triangle(X) \rightarrow \mathbb{R}$ 
is lower semicontinuous (see, e.g., Aliprantis and Border, 2006, Theorem 15.5). Consequently, $\underline{F}_{\Gamma(G)}\left(\bar{d}, \mu^{\prime}\right)>F_{\Gamma(G)}(d, \mu)-\frac{\varepsilon^{*}}{2}>0$ for all $\mu^{\prime}$ in some open neighborhood $\mathcal{N}_{\triangle(X)}(\mu)$ of $\mu$. Since $F_{G}(\bar{d}, x) \geq \underline{F}_{G}(\bar{d}, x)$ for all $x \in X$, we conclude that $F_{\Gamma(G)}\left(\bar{d}, \mu^{\prime}\right) \geq \underline{F}_{\Gamma(G)}\left(\bar{d}, \mu^{\prime}\right)>0$ for all $\mu^{\prime} \in \mathcal{N}_{\triangle(X)}(\mu)$, which means that $\Gamma(G)$ is diagonally transfer continuous.

\section{Proof of Lemma 3}

Fix some $\mu=\left(\mu_{1}, \ldots, \mu_{n}\right) \in \triangle(X), \varepsilon>0$, and $i \in I$. We have to show that, for each $i \in I$, there are a strategy $\sigma_{i} \in \triangle\left(X_{i}\right)$ and a neighborhood $\mathcal{N}_{\triangle\left(X_{-i}\right)}\left(\mu_{-i}\right)$ such that $U_{i}\left(\sigma_{i}, \mu_{-i}^{\prime}\right) \geq u_{i}(\mu)-\varepsilon$ for all $\mu_{-i}^{\prime} \in \mathcal{N}_{\triangle\left(X_{-i}\right)}\left(\mu_{-i}\right)$.

Fix some $i \in I$. There is $x_{i} \in X_{i}$ such that $U_{i}\left(x_{i}, \mu_{-i}\right)>u_{i}(\mu)-\frac{\varepsilon}{4}$. Since $G$ is a bounded game, there exists $B>1$ such that $\left|u_{i}(x)\right|<B$ for every $x \in X$. Pick $\sigma_{i}\left(x_{i}, \mu_{-i}, \frac{\varepsilon}{4 B}\right) \in \triangle\left(X_{i}\right)$ and a Borel set $Q_{-i} \subset X_{-i}$ with $\mu_{-i}\left(Q_{-i}\right)>1-\frac{\varepsilon}{4 B}$ such that $\liminf _{w_{-i} \rightarrow x_{-i}} U_{i}\left(\sigma_{i}\left(x_{i}, \mu_{-i}, \frac{\varepsilon}{4 B}\right), w_{-i}\right) \geq$ $u_{i}\left(x_{i}, x_{-i}\right)-\frac{\varepsilon}{4 B}$ for every $x_{-i} \in Q_{-i}$. For the brevity of notation, denote $\lim \inf _{w_{-i} \rightarrow x_{-i}} U_{i}\left(\sigma_{i}\left(x_{i}, \mu_{-i}, \frac{\varepsilon}{4 B}\right), w_{-i}\right)$ by $\underline{U}_{i}\left(\sigma_{i}\left(x_{i}, \mu_{-i}, \frac{\varepsilon}{4 B}\right), x_{-i}\right)$. Then

$$
\begin{gathered}
\int \underline{U}_{i}\left(\sigma_{i}\left(x_{i}, \mu_{-i}, \frac{\varepsilon}{4 B}\right), x_{-i}\right) d \mu_{-i} \geq \int_{Q_{-i}} \underline{U}_{i}\left(\sigma_{i}\left(x_{i}, \mu_{-i}, \frac{\varepsilon}{4 B}\right), x_{-i}\right) d \mu_{-i}-\frac{\varepsilon}{4} \\
\geq \int_{Q_{-i}} u_{i}\left(x_{i}, x_{-i}\right) d \mu_{-i}-\frac{\varepsilon}{2} \geq U_{i}\left(x_{i}, \mu_{-i}\right)-\frac{3 \varepsilon}{4}>U_{i}(\mu)-\varepsilon
\end{gathered}
$$

The lower semicontinuity of $\underline{U}_{i}\left(\sigma_{i}\left(x_{i}, \mu_{-i}, \frac{\varepsilon}{4 B}\right), \cdot\right)$ implies that $\underline{U}_{i}\left(\sigma_{i}\left(x_{i}, \mu_{-i}, \frac{\varepsilon}{4 B}\right), \cdot\right)$ : $\triangle\left(X_{-i}\right) \rightarrow \mathbb{R}$ defined by $\underline{U}_{i}\left(\sigma_{i}\left(x_{i}, \mu_{-i}, \frac{\varepsilon}{4 B}\right), \mu_{-i}\right)=\int \underline{U}_{i}\left(\sigma_{i}\left(x_{i}, \mu_{-i}, \frac{\varepsilon}{4 B}\right), x_{-i}\right) d \mu_{-i}$ is lower semicontinuous in the second argument (see, e.g., Aliprantis and Border, 2006, Theorem 15.5). Consequently, $\underline{U}_{i}\left(\sigma_{i}\left(x_{i}, \mu_{-i}, \frac{\varepsilon}{4 B}\right), \mu_{-i}^{\prime}\right)>U_{i}(\mu)-\varepsilon$ for all $\mu_{-i}^{\prime}$ in some open neighborhood $\mathcal{N}_{\triangle\left(X_{-i}\right)}\left(\mu_{-i}\right)$ of $\mu_{-i}$. On the other hand, since $U_{i}\left(\sigma_{i}\left(x_{i}, \mu_{-i}, \frac{\varepsilon}{4 B}\right), \mu_{-i}^{\prime}\right) \geq \int \underline{U}_{i}\left(\sigma_{i}\left(x_{i}, \mu_{-i}, \frac{\varepsilon}{4 B}\right), x_{-i}\right) d \mu_{-i}^{\prime}$ for every $\mu_{-i}^{\prime} \in \triangle\left(X_{-i}\right)$, we have that $U_{i}\left(\sigma_{i}\left(x_{i}, \mu_{-i}, \frac{\varepsilon}{4 B}\right), \mu_{-i}^{\prime}\right)>U_{i}(\mu)-\varepsilon$ for every $\mu_{-i}^{\prime} \in \mathcal{N}_{\triangle\left(X_{-i}\right)}\left(\mu_{-i}\right)$, which completes the proof. 


\section{An Auxiliary Lemma}

Lemma 5 Let $X=[0,1] \times[0,1]$, and $\mu_{i} \in \triangle([0,1]), i=1,2$, and let $D$ be a Borel subset of $[0,1]$. Then the set $\bar{D}=\{(z, z) \in[0,1] \times[0,1]: z \in D\}$ is a null set with respect to the product measure $\mu=\mu_{1} \times \mu_{2}$ iff $\mu(x)=0$ for every $x \in \bar{D}$.

Proof. Assume that $\mu(x)=0$ for every $x \in \bar{D}$. We have to show that $\mu(\bar{D})=0$.

First consider the case where, for some $i \in\{1,2\}, \mu_{i}(x)=0$ for all $x \in D$. Fix some arbitrary $\varepsilon>0$. Since $\mu_{i}$ has no mass points on $D$, there exists a finite disjoint collection $\left\{D_{k}\right\}_{k=1}^{m}$ of subsets of $D$ such that $D=\cup_{k=1}^{m} D_{k}$ and $\mu_{i}\left(D_{k}\right) \leq \varepsilon$ for all $k$. Then $\mu(\bar{D}) \leq \sum_{k} \mu\left(D_{k} \times D_{k}\right)=\sum_{k} \mu_{i}\left(D_{k}\right) \mu_{-i}\left(D_{k}\right) \leq$ $\varepsilon \mu_{-i}(D) \leq \varepsilon$, which implies that $\mu(\bar{D})=0$.

Let both $\mu_{1}$ and $\mu_{2}$ have mass points on $D$. Each $\mu_{i}$, as a finite measure, can have at most a countable number of mass points. Denote by $C_{i}$ the set of mass points of $\mu_{i}$ on $D$. Since $\mu(x)=0$ for every $x \in \bar{D}, C_{1} \cap C_{2}=\varnothing$.

Fix some arbitrary $\varepsilon>0$. Since $C_{1} \cap C_{2}=\varnothing$, it is possible to associate with every $x \in C_{1}$ a Borel set $D(x) \subset[0,1]$ containing $x$ such that $\mu_{1}(D(x)) \leq$ $2 \mu_{1}(x)$ and $\mu_{2}(D(x)) \leq \frac{\varepsilon}{2}$. Let $D_{1}=D \backslash C_{1}$. The set $D_{1}$ is Borel, and $\mu_{1}$ is nonatomic on it. Denote by $\bar{C}_{1}\left(\bar{D}_{1}\right)$ the set $\{(z, z) \in[0,1] \times[0,1]: z \in$ $\left.C_{1}\left(z \in D_{1}\right)\right\}$. It is clear that $\bar{C}_{1}$ is at most countable and $\mu\left(\bar{D}_{1}\right)=0$. Then, since $\bar{D}=\bar{C}_{1} \cup \bar{D}_{1}$ and $\bar{C}_{1} \cap \bar{D}_{1}=\varnothing$, we have that $\mu(\bar{D})=\mu\left(\bar{C}_{1}\right)+\mu\left(\bar{D}_{1}\right)=$ $\mu\left(\bar{C}_{1}\right) \leq \sum_{x \in C_{1}} \mu_{1}(D(x)) \mu_{2}(D(x)) \leq \sum_{x \in C_{1}} 2 \mu_{1}(x) \frac{\varepsilon}{2} \leq \varepsilon$.

\section{Some Comments on Theorem 5b of Dasgupta and Maskin (1986)}

It is difficult to overestimate the influence of Theorem 5b of Dasgupta and Maskin (1986) on the subsequent development of equilibrium existence theory. As was pointed out by Bagh (2010), the proof of Theorem 5b requires stronger assumptions than those made initially by Dasgupta and Maskin 
(1986). In what follows we show that the modifications needed are almost impalpable.

Consider a two-player game $G=\left(X_{i}, u_{i}\right)_{i \in\{1,2\}}$ on the unit square, $X=$ $X_{1} \times X_{2}=[0,1] \times[0,1]$, where the payoff functions' discontinuities lie on the main diagonal of $X$. Let $S^{1}=\left\{\left(x_{1}, x_{2}\right) \in[0,1] \times[0,1]: x_{2}>x_{1}\right\}, S^{2}=$ $\left\{\left(x_{1}, x_{2}\right) \in[0,1] \times[0,1]: x_{1}>x_{2}\right\}$, and $S=\left\{\left(x_{1}, x_{2}\right):[0,1] \times[0,1]: x_{1}=x_{2}\right\}$. Denote the set of points at which the sum of the payoff functions is not upper semicontinuous by $S_{D}$; that is, $S_{D}=\left\{x \in X: \limsup _{y \rightarrow x} A_{G}^{0}(y)>A_{G}^{0}(x)\right\}$.

Theorem 7 Consider a two-player game $G=\left(X_{i}, u_{i}\right)_{i \in\{1,2\}}$ on the unit square $X=[0,1] \times[0,1]$. Assume that

(i) there are continuous functions $f_{i}^{j}: \operatorname{cl} S^{j} \rightarrow \mathbb{R}, i=1,2, j=1,2$ such that $u_{i}(x)=f_{i}^{j}(x)$ for all $x \in S^{j}$ and all $i, j \in\{1,2\}$;

(ii) for each $i \in\{1,2\}$ and every $x \in S$, there exists $j \in\{1,2\}$ such that

$$
f_{i}^{j}(x) \geq u_{i}(x) \geq f_{i}^{-j}(x)
$$

(iii) for every point $x=(z, z) \in S_{D}$, there exist $i, j \in\{1,2\}$ such that $\lim _{k \rightarrow \infty} f_{i}^{j}\left(x_{i}^{k}, z\right)>u_{i}(x)$ for some sequence $\left\{\left(x_{i}^{k}, z\right)\right\} \subset S^{j}$ converging to $x$; (iv) if $f_{i}^{j}(x)>u_{i}(x)$ for some $x \in S_{D}$ and $i, j \in\{1,2\}$, then $f_{-i}^{j}(x)<u_{-i}(x)$; (v) $A_{G}^{0}(x) \leq \max _{j \in\{1,2\}}\left\{f_{1}^{j}(x)+f_{2}^{j}(x)\right\}$ for every $x \in S$.

Then $G$ has a mixed strategy Nash equilibrium.

One can check that (i) and (ii) imply that both the initial game and the auxiliary game constructed in the proof are uniformly payoff secure. Condition (iii) is slightly stronger than the assumption that for every point $x=(z, z) \in S_{D}$, there exist $i, j \in\{1,2\}$ such that $f_{i}^{j}(x)>u_{i}(x)$. The difference between them pertains to the points $(0,0)$ and $(1,1)$ only. The strengthening is needed to make it impossible for an equilibrium product measure of the modified game to have mass points at $(0,0)$ or $(1,1)$.

Proof. Define $A_{G}^{0}: X \rightarrow \mathbb{R}$ by $A_{G}^{0}(x)=u_{1}(x)+u_{2}(x)$ for every $x \in X$. It is not difficult to see that $S_{D}=\left\{x \in S: \max _{j \in\{1,2\}}\left(f_{1}^{j}(x)+f_{2}^{j}(x)\right)>A_{G}^{0}(x)\right\}$ 
is a Borel set in $X$.

The proof follows the general lines of Theorem 5b of Dasgupta and Maskin (1986). First we will modify payoffs on $S_{D}$ so as to make the sum of the payoff functions upper semicontinuous on $X$. However, at every $x \in S_{D}$ we will modify only one player's payoff, without changing the other player's payoff. It is possible because if $x \in S_{D}$, then, for some $j(x) \in\{1,2\}, \sum_{i \in\{1,2\}} f_{i}^{j(x)}(x) \geq \sum_{i \in\{1,2\}} f_{i}^{-j(x)}(x)$; that is, in the modified game, the equality $\sum_{i \in\{1,2\}} f_{i}^{j(x)}(x)=A_{\widehat{G}}^{0}(x)$ will imply that $A_{\widehat{G}}^{0}(x) \geq$ $\sum_{i \in\{1,2\}} f_{i}^{-j(x)}(x)$.

If for $x \in S_{D}, \sum_{i \in\{1,2\}} f_{i}^{j}(x)>\sum_{i \in\{1,2\}} f_{i}^{-j}(x)$ for some $j \in\{1,2\}$, then put $j(x)=j$, and, if $\sum_{i \in\{1,2\}} f_{i}^{1}(x)=\sum_{i \in\{1,2\}} f_{i}^{2}(x)$, pick $j(x)$ such that $f_{1}^{j(x)}(x)>u_{1}(x)$. Denote by $i(x)$ the index $i$ such that $f_{i}^{j(x)}(x)>u_{i}(x)$.

Define the modified payoff functions as follows: for any $x \in X \backslash S_{D}$, $\widehat{u}_{i}(x)=u_{i}(x)$ for $i \in\{1,2\}$; for any $x \in S_{D}, \widehat{u}_{i}(x)=u_{i}(x)$ for $i \in$ $\{1,2\} \backslash\{i(x)\}$ and

$\widehat{u}_{i(x)}(x)=f_{i(x)}^{j(x)}(x)-\left(u_{-i(x)}(x)-f_{-i(x)}^{j(x)}(x)\right)=\max _{j \in\{1,2\}}\left(f_{1}^{j}(x)+f_{2}^{j}(x)\right)-u_{-i(x)}(x)$.

Let us show, for example, that $\widehat{u}_{1}$ is Borel measurable on $X$. Denote $S_{i D}=\left\{x \in S_{D}: i(x)=i\right\}, i=1,2$. It is clear that $S_{D}=S_{1 D} \cup S_{2 D}$. The set $S_{1 D}$, in its turn, consists of the following three subsets: $S_{1 D}^{0}=\{x \in$ $\left.S_{D}: \sum_{i \in\{1,2\}} f_{i}^{1}(x)=\sum_{i \in\{1,2\}} f_{i}^{2}(x)\right\}, S_{1 D}^{1}=\left\{x \in S_{D}: \sum_{i \in\{1,2\}} f_{i}^{1}(x)>\right.$ $\sum_{i \in\{1,2\}} f_{i}^{2}(x)$ and $\left.f_{1}^{1}(x)>u_{1}(x)\right\}$, and $S_{1 D}^{2}=\left\{x \in S_{D}: \sum_{i \in\{1,2\}} f_{i}^{2}(x)>\right.$ $\sum_{i \in\{1,2\}} f_{i}^{1}(x)$ and $\left.f_{1}^{2}(x)>u_{1}(x)\right\}$. Thus, $S_{1 D}$ is a Borel set, which, in particular, implies that $\widehat{u}_{1}$ is a Borel measurable function. Clearly, $\widehat{u}_{2}$ is Borel measurable on $X$ as well.

It is useful to notice that $\widehat{u}_{1}(x)+\widehat{u}_{2}(x)=\max _{j \in\{1,2\}}\left(f_{1}^{j}(x)+f_{2}^{j}(x)\right)$ for every $x \in S$. As a result, the function $A_{\widehat{G}}^{0}$ is upper semicontinuous on $X$.

By Lemma 2 and Theorem 3, $\widehat{G}$ has a mixed strategy Nash equilibrium, $\widehat{\mu}=\left(\widehat{\mu}_{1}, \widehat{\mu}_{2}\right)$.

Let us show that $\widehat{\mu}\left(S_{D}\right)=0$. Assume, by way of contradiction, that 
$\widehat{\mu}\left(S_{D}\right)>0$. Since the set $S_{D}$ is Borel and lies on the main diagonal of the unit square, it is clear that the set $\left\{z \in[0,1]:(z, z) \in S_{D}\right\}$ is Borel (see also Kechris, 1995, Theorem 15.1). Then, by Lemma $5, \widehat{\mu}\left(S_{D}\right)>0$ iff $\widehat{\mu}(x)>0$ for some $x=(z, z) \in S_{D}$. That is, $\widehat{\mu}_{i}(z)>0$ for each $i \in\{1,2\}$. By (iii), there exist $i \in\{1,2\}$ and some sequence $\left\{\left(x_{i}^{k}, z\right)\right\} \subset S^{j(x)}$ converging to $x$ such that $\lim _{n \rightarrow \infty} f_{i}^{j(x)}\left(x_{i}^{k}, z\right)>\widehat{u}_{i}(x)$. Then, it is not difficult to see that for every $\varepsilon>0$, there exists $\delta(\varepsilon) \in \mathbb{R} \backslash\{0\}$ such that $z+\delta(\varepsilon) \in[0,1]$ and, for every $d_{i} \in(z, z+\delta(\varepsilon)]$, $\widehat{u}_{i}\left(d_{i}, x_{-i}\right)>\widehat{u}_{i}\left(z, x_{-i}\right)-\varepsilon$ for all $x_{-i} \in X_{-i}$.

Since $\widehat{\mu}_{i}(z)>0, \widehat{U}_{i}\left(\delta_{z}^{D}, \widehat{\mu}_{-i}\right)=\max _{\mu_{i} \in \Delta\left(X_{i}\right)} \widehat{U}_{i}\left(\mu_{i}, \widehat{\mu}_{-i}\right)$. On the other hand, $\widehat{U}_{i}\left(\delta_{z}^{D}, \widehat{\mu}_{-i}\right)=\widehat{\mu}_{-i}(z) u_{i}(z, z)+\int_{[0,1] \backslash\{z\}} \widehat{u}_{i}\left(z, x_{-i}\right) d \widehat{\mu}_{-i}$. Fix some $\varepsilon \in$ $\left(0, \frac{1}{2} \widehat{\mu}_{-i}(z)\left(f_{i}^{j(x)}(z, z)-\widehat{u}_{i}(z, z)\right)\right)$. Pick some $d_{i} \in(z, z+\delta(\varepsilon)]$ such that $\widehat{u}_{i}\left(d_{i}, z\right)=f_{i}^{j(x)}\left(d_{i}, z\right)>\frac{1}{2}\left(f_{i}^{j(x)}(z, z)+\widehat{u}_{i}(z, z)\right)$. Then $\widehat{U}_{i}\left(\delta_{d_{i}}^{D}, \widehat{\mu}_{-i}\right)-\widehat{U}_{i}\left(\delta_{z}^{D}, \widehat{\mu}_{-i}\right)>$ $\widehat{\mu}_{-i}(z)\left(\widehat{u}_{i}\left(d_{i}, z\right)-\widehat{u}_{i}(z, z)\right)-\varepsilon>0$, a contradiction.

Therefore, $\widehat{U}_{i}(\widehat{\mu})=U_{i}(\widehat{\mu}), i=1,2$. Since $\widehat{\mu}$ is a mixed strategy equilibrium of $\widehat{G}$ and, by construction, $\widehat{U}_{i}(\mu) \geq U_{i}(\mu)$ for each $i \in\{1,2\}$ and every $\mu \in \triangle(X)$, we conclude that $\widehat{\mu}$ is also a mixed strategy Nash equilibrium of G.

Results similar to Theorem 7 can also be shown using Simon and Zame's (1990) endogeneous sharing rule approach, which was applied by Siegel (2009) to all-pay contests and by Klose and Kovenock (2013) to all-pay auctions with complete information and identity-dependent externalities.

\section{Proof of Theorem 5}

For a given $\delta>0$ and $z \in[0,1]$, let $\widetilde{C}_{\delta}(z)$ denote the set of all $z^{\prime} \in[0,1]$ such that $\left|z^{\prime}-z\right| \leq \frac{\delta}{2}$. For $x=\left(x_{1}, x_{2}\right) \in[0,1] \times[0,1]$, let $C_{\delta}(x)=\widetilde{C}_{\delta}\left(x_{1}\right) \times \widetilde{C}_{\delta}\left(x_{2}\right)$. Denote by $\mathcal{B}_{[0,1]}$ the $\sigma$-algebra of Borel sets on $[0,1]$. For every $\nu \in \triangle([0,1])$ and a Borel set $Y \subset[0,1]$, denote by $\nu_{\mid Y}$ the restriction of the probability measure $\nu$ to $Y$; that is, $\nu_{\mid Y}(A)=\nu(Y \cap A)$ for every $A \in \mathcal{B}_{[0,1]}$. If $\nu(Y)>0$, then denote by $\bar{\nu}_{\mid Y}$ the probability measure defined by $\bar{\nu}_{\mid Y}(A)=\frac{\nu(Y \cap A)}{\nu(Y)}$ for 
every $A \in \mathcal{B}_{[0,1]}$.

We will show that every game on the unit square satisfying (i)-(iv) has a weakly reciprocally upper semicontinuous mixed extension. Consider a sequence of $\mu^{k}=\left(\mu_{1}^{k}, \mu_{2}^{k}\right) \in \Delta(X)$ converging weakly to $\widehat{\mu}=\left(\widehat{\mu}_{1}, \widehat{\mu}_{2}\right) \in \Delta(X)$ such that $\lim _{k} U_{m}\left(\mu^{k}\right), m=1,2$, exist and $\lim _{k} U_{i}\left(\mu^{k}\right)>U_{i}(\widehat{\mu})$ for some $i \in\{1,2\}$. We have to show that there exist $m \in\{1,2\}$ and $\mu_{m}^{\prime} \in \Delta\left(X_{m}\right)$ such that $U_{m}\left(\mu_{m}^{\prime}, \widehat{\mu}_{-m}\right)>\lim _{k} U_{m}\left(\mu^{k}\right)$.

Since $U_{i}$ jumps down at $\widehat{\mu}$ along the sequence $\left\{\mu^{k}\right\}$, it must be the case that $\widehat{\mu}(S)>0$. Let us first consider the case where each $\widehat{\mu}_{m}$ has a finite number of mass points. Denote by $W(\widehat{\mu})=\left\{y^{1}, \ldots, y^{L}\right\}$ the set of mass points of $\widehat{\mu}$ belonging to $S$, where $y^{s}=\left(z^{s}, z^{s}\right), s=1, \ldots, L$. By Lemma $5, \widehat{\mu}(S)=\sum_{y \in W(\widehat{\mu})} \widehat{\mu}(y)$. Then, for some small enough $\widehat{\delta}_{1}>0$, $C_{\widehat{\delta}_{1}}\left(y^{s}\right) \cap C_{\widehat{\delta}_{1}}\left(y^{t}\right)=\varnothing$ for all $s, t \in\{1, \ldots, L\}, s \neq t$, and, for each $s \in$ $\{1, \ldots, L\}, \widehat{\mu}_{m}(\{z\})=0, m=1,2$, for every $z \in \widetilde{C}_{\widehat{\delta}_{1}}\left(z^{s}\right) \backslash\left\{z^{s}\right\}$. In particular, the latter implies that $\widehat{\mu}\left(\partial C_{\widehat{\delta}_{1}}\left(y^{s}\right)\right)=0$ for each $s \in\{1, \ldots, L\}$. Assume also that $\lim _{k} U_{m}\left(\mu_{m \mid \widetilde{C}_{\widehat{\delta}_{1}}\left(z_{s}\right)}^{k}, \mu_{-m}^{k}\right)$ exists for each $s \in\{1, \ldots, L\}$ and each $m \in\{1,2\}$; if not, consider a subsequence of $\left\{\mu^{k}\right\}$ possessing this property.

Let $y^{1} \in W(\widehat{\mu})$ be such that $\lim _{k} \int_{C_{\widehat{\delta}_{1}}\left(y^{1}\right)} u_{i} d \mu^{k}-\int_{C_{\widehat{\delta}_{1}}\left(y^{1}\right)} u_{i} d \widehat{\mu}>a>0$. Then there exist $j_{1} \in\{1,2\}$ and a subsequence of $\left\{\mu^{k}\right\}$, denoted again by $\left\{\mu^{k}\right\}$, such that $f_{i}^{j_{1}}\left(y^{1}\right)>u_{i}\left(y^{1}\right)$ and $\lim _{k} \mu^{k}\left(C_{\widehat{\delta}_{1}}\left(y^{1}\right) \cap S^{j_{1}}\right)>\widehat{\mu}\left(C_{\widehat{\delta}_{1}}\left(y^{1}\right) \cap S^{j_{1}}\right)$. The reciprocal upper semicontinuity of $G$ implies that $f_{-i}^{j_{1}}\left(y^{1}\right)<u_{-i}\left(y^{1}\right)$. Define

$$
b_{-i}=\frac{1}{2}\left(u_{-i}\left(y^{1}\right)-f_{-i}^{j_{1}}\left(y^{1}\right)\right)\left(\lim _{k} \mu^{k}\left(C_{\widehat{\delta}_{1}}\left(y^{1}\right) \cap S^{j_{1}}\right)-\widehat{\mu}\left(C_{\widehat{\delta}_{1}}\left(y^{1}\right) \cap S^{j_{1}}\right)\right) .
$$

Since all $f_{i}^{j}, i, j \in\{1,2\}$, and $u_{i \mid S}, i \in\{1,2\}$, are uniformly continuous on their respective compact domains $\operatorname{cl} S^{j}$ and $S$, for every $\varepsilon>0$ there exists $\delta(\varepsilon) \in\left(0, \frac{\widehat{\delta}_{1}}{2}\right]$ such that, for all $i, j \in\{1,2\},\left|f_{i}^{j}\left(x^{\prime}\right)-f_{i}^{j}\left(x^{\prime \prime}\right)\right|<\varepsilon$ for all $x^{\prime}$ and $x^{\prime \prime}$ in $\operatorname{cl} S^{j}$ with $\left\|x^{\prime}-x^{\prime \prime}\right\|<2 \delta(\varepsilon)$ and $\left|u_{i \mid S}\left(x^{\prime}\right)-u_{i \mid S}\left(x^{\prime \prime}\right)\right|<\varepsilon$ for all $x^{\prime}$ and $x^{\prime \prime}$ in $S$ with $\left\|x^{\prime}-x^{\prime \prime}\right\|<2 \delta(\varepsilon)$. 
It follows from the uniform payoff security of $G$ that, for each $s \in$ $\{1, \ldots, L\}$, there exists $d_{-i}\left(z_{s}, \frac{b_{-i}}{2^{s+2}}\right) \in X_{-i}$ such that for every $x_{i} \in X_{i}$, $u_{-i}\left(d_{-i}\left(z^{s}, \frac{b_{-i}}{2^{s+2}}\right), w_{i}\right)>u_{-i}\left(z^{s}, x_{i}\right)-\frac{b_{-i}}{2^{s+2}}$ for all $w_{i}$ in some neighborhood $\mathcal{N}_{X_{i}}\left(x_{i}\right)$ of $x_{i}$ in $X_{i}$. For each $s \in\{1, \ldots, L\}$, pick some $\widetilde{\delta}_{s} \in\left(0, \delta\left(\frac{b_{-i}}{2^{s+2}}\right)\right]$ such that $B_{X_{i}}\left(z^{s}, \widetilde{\delta}_{s}\right) \subset \mathcal{N}_{X_{i}}\left(z^{s}\right)$.

We want to show that there exist $d_{-i}^{1} \in X_{-i}$ and $\delta_{1} \in\left(0, \widetilde{\delta}_{1}\right]$ such that $U_{-i}\left(d_{-i}^{1}, \widehat{\mu}_{i}\right)>\lim _{k} U_{-i}\left(\bar{\mu}_{-i \mid \widetilde{C}_{\delta_{1}}\left(z^{1}\right)}^{k}, \mu_{i}^{k}\right)+\frac{b_{-i}}{2 \widehat{\mu}_{-i}\left(\widetilde{C}_{\delta_{1}}\left(z^{1}\right)\right)}$. Consider first the case where $y^{1} \in S \backslash\{(0,0) \cup(1,1)\}$. Put $d_{-i}^{1}=d_{-i}\left(z^{1}, \frac{b_{-i}}{8}\right)$ and $\delta_{1}=\widetilde{\delta}_{1}$. Notice that $U_{-i}\left(d_{-i}^{1}, \widehat{\mu}_{i}\right)=\int_{X_{i} \backslash \widetilde{C}_{\delta_{1}}\left(z^{1}\right)} u_{-i}\left(d_{-i}^{1}, x_{i}\right) d \widehat{\mu}_{i}+\int_{\widetilde{C}_{\delta_{1}}\left(z^{1}\right)} u_{-i}\left(d_{-i}^{1}, x_{i}\right) d \widehat{\mu}_{i}$. Since $u_{-i}\left(d_{-i}^{1}, x_{i}\right)>u_{-i}\left(x_{-i}, x_{i}\right)-\frac{b_{-i}}{2}$ for every $\left(x_{-i}, x_{i}\right) \in \widetilde{C}_{\delta_{1}}\left(z^{1}\right) \times\left(X_{i} \backslash \widetilde{C}_{\delta_{1}}\left(z^{1}\right)\right)$,

$$
U_{-i}\left(d_{-i}^{1}, \widehat{\mu}_{i \mid X_{i} \backslash \widetilde{C}_{\delta_{1}}\left(z^{1}\right)}\right)>U_{-i}\left(\overline{\widehat{\mu}}_{-i \mid \widetilde{C}_{\delta_{1}}\left(z^{1}\right)}, \widehat{\mu}_{i \mid X_{i} \backslash \widetilde{C}_{\delta_{1}}\left(z^{1}\right)}\right)-\widehat{\mu}_{i}\left(X_{i} \backslash \widetilde{C}_{\delta_{1}}\left(z^{1}\right)\right) \frac{b_{-i}}{2}
$$

Since $\widehat{\mu}_{i}\left(\partial \widetilde{C}_{\delta_{1}}\left(z^{1}\right)\right)=\widehat{\mu}_{-i}\left(\partial \widetilde{C}_{\delta_{1}}\left(z^{1}\right)\right)=0, u_{-i}$ is continuous on $\widetilde{C}_{\delta_{1}}\left(z^{1}\right) \times$ $\left(X_{i} \backslash \widetilde{C}_{\delta_{1}}\left(z^{1}\right)\right)$, and the sequence $\left\{\left(\bar{\mu}_{-i \mid \widetilde{C}_{\delta_{1}}\left(z^{1}\right)}^{k}, \mu_{i \mid X_{i} \backslash \widetilde{C}_{\delta_{1}}\left(z^{1}\right)}^{k}\right)\right\}$ converges weakly to $\left(\widehat{\widehat{\mu}}_{-i \mid \widetilde{C}_{\delta_{1}}\left(z^{1}\right)}, \widehat{\mu}_{i \mid X_{i} \backslash \widetilde{C}_{\delta_{1}}\left(z^{1}\right)}\right)$, we have that

$$
U_{-i}\left(\widehat{\mu}_{-i \mid \widetilde{C}_{\delta_{1}}\left(z^{1}\right)}, \widehat{\mu}_{i \mid X_{i} \backslash \widetilde{C}_{\delta_{1}}\left(z^{1}\right)}\right)=\lim _{k} U_{-i}\left(\bar{\mu}_{-i \mid \widetilde{C}_{\delta_{1}}\left(z^{1}\right)}^{k}, \mu_{i \mid X_{i} \backslash \widetilde{C}_{\delta_{1}}\left(z^{1}\right)}^{k}\right) .
$$

Therefore,

$U_{-i}\left(d_{-i}^{1}, \widehat{\mu}_{i \mid X_{i} \backslash \widetilde{C}_{\delta_{1}}\left(z^{1}\right)}\right)>\lim _{k} U_{-i}\left(\bar{\mu}_{-i \mid \widetilde{C}_{\delta_{1}}\left(z^{1}\right)}^{k}, \mu_{i \mid X_{i} \backslash \widetilde{C}_{\delta_{1}}\left(z^{1}\right)}^{k}\right)-\widehat{\mu}_{i}\left(X_{i} \backslash \widetilde{C}_{\delta_{1}}\left(z^{1}\right)\right) \frac{b_{-i}}{2}$

A further important fact is that $u_{-i}\left(d_{-i}^{1}, x_{i}\right)>\sup _{y \in \widetilde{C}_{\delta_{1}}\left(z^{1}\right) \times \widetilde{C}_{\delta_{1}}\left(z^{1}\right)} u_{-i}(y)-$ $\frac{b_{-i}}{2}$ for every $x_{i} \in \widetilde{C}_{\delta_{1}}\left(z^{1}\right)$. To understand it, notice that for some $z_{i} \in \widetilde{C}_{\delta_{1}}\left(z^{1}\right)$,

$$
u_{-i}\left(z^{1}, z_{i}\right)>\sup _{y \in \widetilde{C}_{\delta_{1}}\left(z^{1}\right) \times \widetilde{C}_{\delta_{1}}\left(z^{1}\right)} u_{-i}(y)-\frac{b_{-i}}{8},
$$

which would not be necessarily true if $y^{1}$ were $(0,0)$ or $(1,1)$. Moreover, $u_{-i}\left(d_{-i}^{1}, z_{i}\right)>u_{-i}\left(z^{1}, z_{i}\right)-\frac{b_{-i}}{8}$, and $u_{-i}\left(d_{-i}^{1}, x_{i}\right)-u_{-i}\left(d_{-i}^{1}, z_{i}\right)>-\frac{b_{-i}}{8}$ for 
every $x_{i}, z_{i} \in \widetilde{C}_{\delta_{1}}\left(z^{1}\right)$.

Then

$$
U_{-i}\left(d_{-i}^{1}, \widehat{\mu}_{i \mid \widetilde{C}_{\delta_{1}}\left(z^{1}\right)}\right)>\widehat{\mu}_{i}\left(\widetilde{C}_{\delta_{1}}\left(z^{1}\right)\right)\left(\sup _{y \in \widetilde{C}_{\delta_{1}}\left(z^{1}\right) \times \widetilde{C}_{\delta_{1}}\left(z^{1}\right)} u_{-i}(y)-\frac{b_{-i}}{2}\right) .
$$

It follows from the definition of $b_{-i}$ that for some subsequence of $\left\{\mu^{k}\right\}$, denoted again by $\left\{\mu^{k}\right\}, \widehat{\mu}_{1}\left(\widetilde{C}_{\delta_{1}}\left(z^{1}\right)\right) \widehat{\mu}_{2}\left(\widetilde{C}_{\delta_{1}}\left(z^{1}\right)\right) \sup _{y \in \widetilde{C}_{\delta_{1}}\left(z^{1}\right) \times \widetilde{C}_{\delta_{1}}\left(z^{1}\right)} u_{-i}(y)-$ $\lim _{k} U_{-i}\left(\mu_{-i \mid \widetilde{C}_{\delta_{1}}\left(z^{1}\right)}^{k}, \mu_{i \mid \widetilde{C}_{\delta_{1}}\left(z^{1}\right)}^{k}\right)>b_{-i}$, and, therefore,

$$
\begin{aligned}
\widehat{\mu}_{i}\left(\widetilde{C}_{\delta_{1}}\left(z^{1}\right)\right) \sup _{y \in \widetilde{C}_{\delta_{1}}\left(z^{1}\right) \times \widetilde{C}_{\delta_{1}}\left(z^{1}\right)} u_{-i}(y) & \\
& >\lim _{k} U_{-i}\left(\frac{\mu_{-i \mid \widetilde{C}_{\delta_{1}}\left(z^{1}\right)}^{k}}{\widehat{\mu}_{-i}\left(\widetilde{C}_{\delta_{1}}\left(z^{1}\right)\right)}, \mu_{i \mid \widetilde{C}_{\delta_{1}}\left(z^{1}\right)}^{k}\right)+\frac{b_{-i}}{\widehat{\mu}_{-i}\left(\widetilde{C}_{\delta_{1}}\left(z^{1}\right)\right)} .
\end{aligned}
$$

Then $U_{-i}\left(d_{-i}^{1}, \widehat{\mu}_{i \mid \widetilde{C}_{\delta_{1}}\left(z^{1}\right)}\right)>\lim _{k} U_{-i}\left(\frac{\mu_{-i \mid \widetilde{C}_{\delta_{1}}\left(z^{1}\right)}^{k}}{\widehat{\mu}_{-i}\left(\widetilde{C}_{\delta_{1}}\left(z^{1}\right)\right)}, \mu_{i \mid \widetilde{C}_{\delta_{1}}\left(z^{1}\right)}^{k}\right)-\widehat{\mu}_{i}\left(\widetilde{C}_{\delta_{1}}\left(z^{1}\right)\right) \frac{b_{-i}}{2}+$ $\frac{b_{-i}}{\widehat{\mu}_{-i}\left(\widetilde{C}_{\delta_{1}}\left(z^{1}\right)\right)}$. Since $\lim _{k} \mu_{-i}^{k}\left(\widetilde{C}_{\delta_{1}}\left(z^{1}\right)\right)=\widehat{\mu}_{-i}\left(\widetilde{C}_{\delta_{1}}\left(z^{1}\right)\right)$, we have that

$$
\lim _{k} U_{-i}\left(\frac{\mu_{-i \mid \widetilde{C}_{\delta_{1}}\left(z^{1}\right)}^{k}}{\widehat{\mu}_{-i}\left(\widetilde{C}_{\delta_{1}}\left(z^{1}\right)\right)}, \mu_{i \mid \widetilde{C}_{\delta_{1}}\left(z^{1}\right)}^{k}\right)=\lim _{k} U_{-i}\left(\bar{\mu}_{-i \mid \widetilde{C}_{\delta_{1}}\left(z^{1}\right)}^{k}, \mu_{i \mid \widetilde{C}_{\delta_{1}}\left(z^{1}\right)}^{k}\right)
$$

and, therefore,

$$
\begin{aligned}
U_{-i}\left(d_{-i}^{1}, \widehat{\mu}_{i}\right)>\lim _{k} U_{-i}\left(\bar{\mu}_{-i \mid \widetilde{C}_{\delta_{1}}\left(z^{1}\right)}^{k}, \mu_{i}^{k}\right) & \\
& -\left(\widehat{\mu}_{i}\left(\widetilde{C}_{\delta_{1}}\left(z^{1}\right)\right)+\widehat{\mu}_{i}\left(X_{i} \backslash \widetilde{C}_{\delta_{1}}\left(z^{1}\right)\right)\right) \frac{b_{-i}}{2}+\frac{b_{-i}}{\widehat{\mu}_{-i}\left(\widetilde{C}_{\delta_{1}}\left(z^{1}\right)\right)}
\end{aligned}
$$

which implies that $U_{-i}\left(d_{-i}^{1}, \widehat{\mu}_{i}\right)>\lim _{k} U_{-i}\left(\bar{\mu}_{-i \mid \widetilde{C}_{\delta_{1}}\left(z^{1}\right)}^{k}, \mu_{i}^{k}\right)+\frac{b_{-i}}{2 \widehat{\mu}_{-i}\left(\widetilde{C}_{\delta_{1}}\left(z^{1}\right)\right)}$.

Consider the case where $y^{1}=(0,0)$. If $-i=1$, then, since $j_{1}=1$ by virtue of (iii), put $d_{1}^{1}=\delta_{1}=\delta\left(\frac{b_{-i}}{8}\right)$. In this case, it is clear that $u_{-i}\left(d_{1}^{1}, x_{i}\right)>\sup _{y \in \widetilde{C}_{\delta_{1}}\left(z^{1}\right) \times \widetilde{C}_{\delta_{1}}\left(z^{1}\right)} u_{-i}(y)-\frac{b_{-i}}{2}$ for every $x_{i} \in \widetilde{C}_{\delta_{1}}(0)$. The 
rest of the argument is quite similar to that provided above. Let $-i=2$. If $j_{1}=1$, then the uniform payoff security condition for player 2's strategy 0 can be made use of. If $j_{1}=2$ and $f_{2}^{1}(0,0) \geq u_{2}(0,0)$, then, put $d_{2}^{1}=\delta_{1}=\delta\left(\frac{b_{-i}}{8}\right)$. If $j_{1}=2$ and $f_{2}^{1}(0,0)<u_{2}(0,0)$, then the uniform payoff security condition for player 2's strategy 0 can be made use of. The case where $y^{1}=(1,1)$ can be handled in a similar manner.

We will now describe how to pick, for each $s \in\{2, \ldots, L\}, d_{-i}^{s} \in X_{-i}$ and $\delta_{s} \in\left(0, \delta\left(\frac{b_{-i}}{2^{s+2}}\right)\right]$ such that $U_{-i}\left(d_{-i}^{s}, \widehat{\mu}_{i}\right)>\lim _{k} U_{-i}\left(\bar{\mu}_{-i \mid \widetilde{C}_{\delta_{s}}\left(z^{s}\right)}^{k}, \mu_{i}^{k}\right)-\frac{b_{-i}}{2^{s} \widehat{\mu}_{-i}\left(\widetilde{C}_{\delta_{s}}\left(z^{s}\right)\right)}$. Let $y^{s} \in X \backslash\{(0,0) \cup(1,1)\}$. If $u_{-i}\left(y^{s}\right)>\min \left\{f_{-i}^{1}\left(y^{s}\right), f_{-i}^{2}\left(y^{s}\right)\right\}$ or $u_{-i}\left(y^{s}\right)=$ $f_{-i}^{1}\left(y^{s}\right)=f_{-i}^{2}\left(y^{s}\right)$, then it is possible to make use of the uniform payoff security condition for player $-i$ 's strategy $z^{s}$. If $y^{s}$ is a point of discontinuity of $u_{-i}$ and $u_{-i}\left(y^{s}\right) \leq f_{-i}^{j}\left(y^{s}\right) \leq f_{-i}^{-j}\left(y^{s}\right)$ for some $j \in\{1,2\}$, then, to avoid ambiguity in notation, denote $l=-i=\{1,2\} \backslash\{i\}$, and put $d_{-i}^{s}=z^{s}+(-1)^{j+l} \delta$ and $\delta_{s}=\delta$ for some $\delta \in\left(0, \delta\left(\frac{b_{-i}}{2^{s+2}}\right)\right]$ such that $d_{-i}^{s} \in[0,1]$.

Assume that $y^{s}=(0,0)$ and $-i=2$. It follows from (iii) that $u_{2}(0,0) \geq$ $f_{2}^{2}(0,0)$. If $f_{2}^{1}(0,0) \geq u_{2}(0,0)$, then put $d_{2}^{s}=\delta_{s}=\delta\left(\frac{b_{-i}}{2^{s+2}}\right)$. If $f_{2}^{1}(0,0)<$ $u_{2}(0,0)$, then the uniform payoff security condition for player 2 's strategy 0 can be made use of.

Assume that $y^{s}=(0,0)$ and $-i=1$. By (iii), $u_{1}(0,0) \leq f_{1}^{2}(0,0)$. If, moreover, $f_{1}^{1}(0,0) \leq f_{1}^{2}(0,0)$, then, put $d_{1}^{s}=\delta_{s}=\delta\left(\frac{b_{-i}}{2^{s+2}}\right)$. If $f_{1}^{1}(0,0)>f_{1}^{2}(0,0)$, then it is important whether there is some subsequence of $\left\{\mu^{k}\right\}$, denoted again by $\left\{\mu^{k}\right\}$, such that $\lim _{k} \mu^{k}\left(C_{\widetilde{\delta}_{1}}(0,0) \cap S^{1}\right)>\widehat{\mu}\left(C_{\widetilde{\delta}_{1}}(0,0) \cap S^{1}\right)$. If this is not the case, then it is possible to choose a small enough $\delta_{s} \in\left(0, \delta\left(\frac{b_{-i}}{2^{s+2}}\right)\right]$ such that $U_{1}\left(\delta_{s}, \widehat{\mu}_{2}\right)>\lim _{k} U_{1}\left(\bar{\mu}_{1 \mid \widetilde{C}_{\delta_{s}}(0)}^{k}, \mu_{2}^{k}\right)-\frac{b_{1}}{2^{s} \widehat{\mu}_{1}\left(\widetilde{C}_{\delta_{s}}(0)\right)}$. This is so because, by choosing $\delta_{s}$ close enough to $0, \widehat{\mu}\left(C_{2 \delta_{s}}(0,0) \cap S^{1}\right)$ can be made arbitrarily small, and, moreover, $G$ is a compact game and $\lim \sup _{k} \mu^{k}\left(C_{2 \delta_{s}}(0,0) \cap S^{1}\right) \leq$ $\widehat{\mu}\left(C_{2 \delta_{s}}(0,0) \cap S^{1}\right)$. However, if such a subsequence of $\left\{\mu^{k}\right\}$ exists, then one of the possible ways to circumvent the obstacle is to repeat the above argument for player $2(-i=2)$, with $y^{1}=(0,0)$ and $j_{1}=1$. A similar problem can not occur in this case even if $y^{s}=(1,1)$ for some $s \geq 2$ by virtue of (iii). 
Then $\left.\lim _{k} U_{-i}\left(\mu_{-i}^{k}, \mu_{i}^{k}\right)=\sum_{s=\overline{1, L}} \widehat{\mu}_{-i}\left(\widetilde{C}_{\delta_{s}}\left(z^{s}\right)\right) \lim _{k} U_{-i}\left(\bar{\mu}_{-i \mid \widetilde{C}_{\delta_{s}}\left(z^{s}\right)}, \mu_{i}^{k}\right)\right)+$ $U_{-i}\left(\widehat{\mu}_{-i \mid X_{-i} \backslash \cup_{s=1, L} \widetilde{C}_{\delta_{s}}\left(z^{s}\right)}, \widehat{\mu}_{i}\right)$, and, consequently, it is not difficult to see that $\lim _{k} U_{-i}\left(\mu_{-i}^{k}, \mu_{i}^{k}\right)<\sum_{s=1, L} \widehat{\mu}_{-i}\left(\widetilde{C}_{\delta_{s}}\left(z^{s}\right)\right) U_{-i}\left(d_{-i}^{s}, \widehat{\mu}_{i}\right)+U_{-i}\left(\widehat{\mu}_{-i \mid X_{-i} \backslash \bigcup_{s=1, L}} \widetilde{C}_{\delta_{s}}\left(z^{s}\right), \widehat{\mu}_{i}\right)$. Define $\mu_{-i}^{\prime} \in \triangle\left(X_{-i}\right)$ as follows:

$$
\mu_{-i}^{\prime}=\sum_{s=\overline{1, L}} \widehat{\mu}_{-i}\left(\widetilde{C}_{\delta_{s}}\left(z^{s}\right)\right) \delta_{d_{-i}^{s}}^{D}+\widehat{\mu}_{-i \mid X-i \backslash \cup_{s=\overline{1, L}}}^{\prime} \widetilde{C}_{\delta_{s}}\left(z^{s}\right) .
$$

Then we have that $\lim _{k} U_{-i}\left(\mu_{-i}^{k}, \mu_{i}^{k}\right)<U_{-i}\left(\mu_{-i}^{\prime}, \widehat{\mu}_{i}\right)$, which implies that $\Gamma$ is weakly reciprocally upper semicontinuous.

The case where each $\widehat{\mu}_{m}$ has a countable number of mass points can be treated similarly because $\widehat{\mu}_{1}$ and $\widehat{\mu}_{2}$ are finite measures and $G$ is a compact game. For example, let the set of mass points of $\widehat{\mu}$ lying on $S, W(\widehat{\mu})$, be a countable set. Since $U_{i}$ jumps down at $\widehat{\mu}$ along the sequence $\left\{\mu^{k}\right\}$, there are $y^{1} \in W(\widehat{\mu})$ and $\widehat{\delta}_{1}>0$ such that $\liminf _{k} \int_{C_{\delta}\left(y^{1}\right)} u_{i} d \mu^{k}-\int_{C_{\delta}\left(y^{1}\right)} u_{i} d \widehat{\mu}>a>0$ for every $\delta \in\left(0, \widehat{\delta}_{1}\right]$. Moreover, for every $\varepsilon>0$ there exists $\delta_{\varepsilon} \in\left(0, \widehat{\delta}_{1}\right]$ such that $\widehat{\mu}\left(\left(W(\widehat{\mu}) \backslash\left\{y_{1}\right\}\right) \cap C_{\delta_{\varepsilon}}\left(y^{1}\right)\right)<\varepsilon$ and $\widehat{\mu}_{i}\left(\partial \widetilde{C}_{\delta_{\varepsilon}}\left(z^{1}\right)\right)=\widehat{\mu}_{-i}\left(\partial \widetilde{C}_{\delta_{\varepsilon}}\left(z^{1}\right)\right)=$ 0 , which makes it possible to repeat the above reasoning for $C_{\delta_{1}}\left(y^{1}\right)$ with some small enough $\delta_{1} \in\left(0, \widehat{\delta}_{1}\right]$. Then pick another mass point from the set $W(\widehat{\mu}) \backslash C_{\delta_{1}}\left(y^{1}\right)$, if there are any left in it. Denote it by $y^{2}$. It is again possible to apply the argument. However, in this case, it may be necessary to consider an infinite number of the mass points of $\widehat{\mu}$ lying on $S$.

\section{Proof of Theorem 6}

For the sake of notational simplicity, denote $X_{h_{1}}^{1}$ by $T^{1}, X_{h_{1}}^{2} \cap X_{h_{2}}^{1}$ by $T^{2}$, and $X_{h_{2}}^{2}$ by $T^{3}$. Then, for each $i \in\{1,2\}, u_{i}(x)=f_{i}^{1}(x)$ for every $x \in T^{1}$, $u_{i}(x)=f_{i}^{2}(x)$ for every $x \in T^{2}$, and $u_{i}(x)=f_{i}^{3}(x)$ for every $x \in T^{3}$.

Consider a sequence of $\mu^{k}=\left(\mu_{1}^{k}, \mu_{2}^{k}\right) \in \Delta(X)$ converging weakly to $\widehat{\mu} \in \Delta(X)$ such that $\lim _{k} U_{i}\left(\mu^{k}\right)>U_{i}(\widehat{\mu})$ for some $i \in\{1,2\}$. Assume, without loss of generality, that each $\widehat{\mu}_{m}$ has a finite number of mass points 
and $\lim _{k} U_{m}\left(\mu^{k}\right), m=1,2$, exist. We have to show that there exist $m \in\{1,2\}$ and $\mu_{m}^{\prime} \in \Delta\left(X_{m}\right)$ such that $U_{m}\left(\mu_{m}^{\prime}, \widehat{\mu}_{-m}\right)>\lim _{k} U_{m}\left(\mu^{k}\right)$.

Since $U_{i}$ jumps down at $\widehat{\mu}$ along the sequence $\left\{\mu^{k}\right\}$, it must be the case that $\widehat{\mu}\left(X_{h_{1}} \cup X_{h_{2}}\right)>0$. Denote by $W(\widehat{\mu})=\left\{y^{1}, \ldots, y^{L}\right\}$, the set of mass points of $\widehat{\mu}$ belonging to $X_{h_{1}} \cup X_{h_{2}}$. Then $\widehat{\mu}\left(X_{h_{1}} \cup X_{h_{2}}\right)=\sum_{s=\overline{1, L}} \widehat{\mu}\left(y^{s}\right)$, and there exists some $\widehat{\delta}_{1}>0$ such that $C_{\widehat{\delta}_{1}}\left(y^{s}\right) \cap C_{\widehat{\delta}_{1}}\left(y^{t}\right)=\varnothing$ for all $s, t \in\{1, \ldots, L\}, s \neq t$. Assume also that $\lim _{k} U_{m}\left(\mu_{m \mid \widetilde{C}_{\widehat{\delta}_{1}}\left(z_{m}^{s}\right)}^{k}, \mu_{-m}^{k}\right)$ exists for each $s \in\{1, \ldots, L\}$ and each $m \in\{1,2\}$. Without loss of generality, $y^{1}=\left(z_{1}^{1}, z_{2}^{1}\right) \in X_{h_{2}}$ and $\lim _{k} \int_{C_{\widehat{\delta}_{1}}\left(y^{1}\right)} u_{i} d \mu^{k}-\int_{C_{\hat{\delta}_{1}}\left(y^{1}\right)} u_{i} d \widehat{\mu}>a>0$.

For some $j_{1} \in\{2,3\}$ and some subsequence of $\left\{\mu^{k}\right\}$, denoted again by $\left\{\mu^{k}\right\}$, we have that $f_{i}^{j_{1}}\left(y^{1}\right)>u_{i}\left(y^{1}\right)$ and $\lim _{k} \mu^{k}\left(C_{\widehat{\delta}_{1}}\left(y^{1}\right) \cap T^{j_{1}}\right)>\widehat{\mu}\left(C_{\widehat{\delta}_{1}}\left(y^{1}\right) \cap\right.$ $\left.T^{j_{1}}\right)$. The reciprocal upper semicontinuity of $G$ implies that $f_{-i}^{j_{1}}\left(y^{1}\right)<$ $u_{-i}\left(y^{1}\right)$. Define

$$
b_{-i}=\frac{1}{2}\left(u_{-i}\left(y^{1}\right)-f_{-i}^{j_{1}}\left(y^{1}\right)\right)\left(\lim _{k} \mu^{k}\left(C_{\widehat{\delta}_{1}}\left(y^{1}\right) \cap T^{j_{1}}\right)-\widehat{\mu}\left(C_{\widehat{\delta}_{1}}\left(y^{1}\right) \cap T^{j_{1}}\right)\right) .
$$

Consider first the case where $-i=2$. Since the details of the argument in this case is similar to those that can be found in the proof of Theorem 5 , we will describe how to choose $d_{2}^{1} \in X_{2}$ and $\delta_{1} \in\left(0, \widehat{\delta}_{1}\right]$ such that $U_{2}\left(d_{2}^{1}, \widehat{\mu}_{1}\right)>$ $\lim _{k} U_{2}\left(\bar{\mu}_{2 \mid \widetilde{C}_{\delta_{1}}\left(z_{2}^{1}\right)}^{k}, \mu_{1}^{k}\right)+\frac{b_{2}}{2 \widehat{\mu}_{2}\left(\widetilde{C}_{\delta_{1}}\left(z_{2}^{1}\right)\right)}$ in a schematic way. First notice that the cases where $y^{1} \in X_{h_{2}} \backslash\left(\left\{\left(0, \frac{1}{4}\right)\right\} \cup\{(1,0)\}\right)$ can be handled with no difficulty through making use of the uniform payoff security condition for player 2's strategy $y_{2}^{1}$.

Let $y^{1}=\left(0, \frac{1}{4}\right)$. If $j_{1}=2$ and $u_{2}\left(0, \frac{1}{4}\right)>f_{2}^{3}\left(0, \frac{1}{4}\right)$ or $j_{1}=3$ and $u_{2}\left(0, \frac{1}{4}\right)>$ $f_{2}^{2}\left(0, \frac{1}{4}\right)$, then one can make use of the uniform payoff security condition for player 2's strategy $\frac{1}{4}$ to find suitable $d_{2}^{1}$ and $\delta_{1}$. If $j_{1}=3$ and $u_{2}\left(0, \frac{1}{4}\right) \leq$ $f_{2}^{2}\left(0, \frac{1}{4}\right)$ or $j_{1}=2$ and $u_{2}\left(0, \frac{1}{4}\right) \leq f_{2}^{3}\left(0, \frac{1}{4}\right)$, then $d_{2}^{1}=\frac{1}{4}+(-1)^{\left(j_{1}-1\right)} \delta_{1}$ with a small enough $\delta_{1} \in\left(0, \widehat{\delta}_{1}\right]$.

Let $y^{1}=(1,0)$. By virtue of (iii), $u_{2}(1,0) \geq f_{2}^{3}(1,0)$. If $j_{1}=2$ and $u_{2}(1,0)>f_{2}^{3}(1,0)$ or $j_{1}=3$ and $u_{2}(1,0)>f_{2}^{2}(1,0)$, then the uniform payoff 
security condition for player 2's strategy 0 can be made use of. If $j_{1}=2$ and $u_{2}(1,0)=f_{2}^{3}(1,0)$ or $j_{1}=3$ and $u_{2}(1,0) \leq f_{2}^{2}(1,0)$, then put $d_{2}^{1}=\left(j_{1}-2\right) \delta_{1}$ with a small enough $\delta_{1} \in\left(0, \widehat{\delta}_{1}\right]$.

We now have to describe how to choose, for each $s \in\{2, \ldots, L\}$, a deviation strategy $d_{2}^{s} \in X_{2}$ and a small enough $\delta_{s}>0$ such that $U_{2}\left(d_{2}^{s}, \widehat{\mu}_{1}\right)>$ $\lim _{k} U_{2}\left(\bar{\mu}_{2 \mid \widetilde{C}_{\delta_{s}}\left(z_{2}^{s}\right)}^{k}, \mu_{1}^{k}\right)-\frac{b_{2}}{2^{s} \widehat{\mu}_{2}\left(\widetilde{C}_{\delta_{s}}\left(z_{2}^{s}\right)\right)}$. It is also done in a schematic way.

Assume, for example, that $y^{s}=\left(0, \frac{1}{4}\right)$ for some $s \in\{2, \ldots, L\}$. If $u_{2}\left(0, \frac{1}{4}\right) \geq \max \left\{f_{2}^{2}\left(0, \frac{1}{4}\right), f_{2}^{3}\left(0, \frac{1}{4}\right)\right\}$, then the uniform payoff security condition for player 2's strategy $\frac{1}{4}$ can be used to find suitable $d_{2}^{s}$ and $\delta_{s}$. Let $u_{2}\left(0, \frac{1}{4}\right)<$ $\max \left\{f_{2}^{2}\left(0, \frac{1}{4}\right), f_{2}^{3}\left(0, \frac{1}{4}\right)\right\}$. If $f_{2}^{2}\left(0, \frac{1}{4}\right) \geq f_{2}^{3}\left(0, \frac{1}{4}\right)\left(f_{2}^{2}\left(0, \frac{1}{4}\right)<f_{2}^{3}\left(0, \frac{1}{4}\right)\right)$, then put $d_{2}^{s}=\frac{1}{4}+\delta_{s}\left(d_{2}^{s}=\frac{1}{4}-\delta_{s}\right)$ with a small enough $\delta_{s} \in\left(0, \widehat{\delta}_{1}\right]$. The cases where $y^{s} \in X_{h_{2}} \backslash\left\{\left(0, \frac{1}{4}\right)\right\}$ for some $s \in\{2, \ldots, L\}$ can be handled in a similar manner. The only case worth mentioning separately is where $y^{s}=(1,0)$ and $f_{2}^{2}(1,0)<f_{2}^{3}(1,0)$. Recalling that $u_{2}(1,0) \geq f_{2}^{3}(1,0)$ by virtue of (iii), put $d_{2}^{s}=0$ and pick a small enough $\delta_{s} \in\left(0, \widehat{\delta}_{1}\right]$. A similar argument can be provided for the mass points of $\widehat{\mu}_{2}$ lying on $X_{h_{1}}$.

Thus, if, for some $s \in\{1, \ldots, L\}$, some $j_{1} \in\{1,2,3\}$, and some subsequence of $\left\{\mu^{k}\right\}$, denoted again by $\left\{\mu^{k}\right\}$, the inequalities $f_{1}^{j_{1}}\left(y^{s}\right)>u_{1}\left(y^{s}\right)$ and $\lim _{k} \mu^{k}\left(C_{\widehat{\delta}_{1}}\left(y^{s}\right) \cap T^{j_{1}}\right)>\widehat{\mu}\left(C_{\widehat{\delta}_{1}}\left(y^{s}\right) \cap T^{j_{1}}\right)$ hold, then it is possible to construct $\mu_{2}^{\prime} \in \triangle\left(X_{2}\right)$ such that $U_{2}\left(\mu_{2}^{\prime}, \widehat{\mu}_{1}\right)>\lim _{k} U_{2}\left(\mu_{2}^{k}, \mu_{1}^{k}\right)$. Assume now that this is not the case; then $-i=1$. We will now show that there exists $\mu_{1}^{\prime} \in \triangle\left(X_{1}\right)$ such that $U_{1}\left(\mu_{1}^{\prime}, \widehat{\mu}_{2}\right)>\lim _{k} U_{1}\left(\mu_{1}^{k}, \mu_{2}^{k}\right)$.

If $j_{1}=2$ and $y^{1}$ is $(1,0)$ or $(1,1)$, then put $d_{1}^{1}=1-\delta_{1}$ with a small enough $\delta_{1} \in\left(0, \widehat{\delta}_{1}\right]$ (here not only (iii) is used but also the assumption that $-i$ can not be equal to 2$)$. If $y^{1} \in\left(X_{h_{1}} \cup X_{h_{2}}\right) \backslash(\{(1,0)\} \cup\{(1,1)\})$, then the uniform payoff security condition for player 1 's strategy $y_{1}^{1}$ can be made use of to find suitable $d_{1}^{1}$ and $\delta_{1}$.

If, for some $s \in\{2, \ldots, L\}, y^{s}=(1,0)$ (or $(1,1)$ ), then $d_{1}^{s}=1-\delta_{s}$ with a small enough $\delta_{s} \in\left(0, \widehat{\delta}_{1}\right]$, which is possible owing to the assumption that $-i$ can not be equal to 2 . Let, for example, $y^{s}=\left(y_{1}^{s}, y_{2}^{s}\right) \in X_{h_{2}} \backslash\{(1,0)\}$ for some 
$s \in\{2, \ldots, L\}$. An attempt to apply the uniform payoff security condition for player 1's strategy $y_{1}^{s}$ may not succeed here, since, in general, it is possible for $d_{1}^{s}$ to coincide with $y_{1}^{s}$. Denote by $y^{d}=\left(y_{1}^{d}, y_{2}^{d}\right)$ the point belonging to $X_{h_{1}}$ such that $y_{1}^{d}=y_{1}^{s}$. If $u_{1}\left(y^{s}\right) \leq f_{1}^{2}\left(y^{s}\right)$ and $u_{1}\left(y^{d}\right) \leq f_{1}^{2}\left(y^{d}\right)$, then $d_{1}^{s}=y_{1}^{s}+\delta_{s}$ with a small enough $\delta_{s} \in\left(0, \widehat{\delta}_{1}\right]$, which is possible since if $f_{1}^{3}\left(y^{s}\right)>u_{1}\left(y^{s}\right)$ $\left(f_{1}^{1}\left(y^{d}\right)>u_{1}\left(y^{d}\right)\right)$, then it must be the case that $\lim \sup _{k} \mu^{k}\left(C_{\delta_{s}}\left(y^{s}\right) \cap T^{3}\right) \leq$ $\widehat{\mu}\left(C_{\delta_{s}}\left(y^{s}\right) \cap T^{3}\right)\left(\limsup _{k} \mu^{k}\left(C_{\delta_{s}}\left(y^{d}\right) \cap T^{1}\right) \leq \widehat{\mu}\left(C_{\delta_{s}}\left(y^{d}\right) \cap T^{1}\right)\right)$. If $u_{1}\left(y^{s}\right)>$ $f_{1}^{2}\left(y^{s}\right)$ or $u_{1}\left(y^{d}\right)>f_{1}^{2}\left(y^{d}\right)$, then the uniform payoff security condition for player 1's strategy $y_{1}^{s}$ can be used to find suitable $d_{1}^{s}$ and $\delta_{s}$.

\section{References}

Aliprantis, C.D, Border, K.C., 2006. Infinite Dimensional Analysis, A Hitchhiker's Guide, 3rd ed. Berlin: Springer-Verlag.

Aliprantis, C.D., Glycopantis, D., Puzzello, D., 2006. The joint continuity of the expected payoff functions. J. Math. Econ. 42, 121-130.

Amir, R., 2005. Ordinal versus cardinal complementarity: The case of Cournot oligopoly. Games and Econ. Behav. 53, 1-14.

Bagh, A., Jofre, A., 2006. Reciprocal upper semicontinuity and better reply secure games: a comment. Econometrica 74, 1715-1751.

Bagh, A., 2010. Variational convergence: approximation and existence of equilibria in discontinuous games, J. Econ. Theory 145, 1244-1268.

Ball, R., 1999. Discontinuity and nonexistence of equilibrium in the probabilistic spatial voting model. Soc. Choice Welfare 16, 533-555.

Barelli, P., Meneghel, I., 2013. A note on the equilibrium existence problem in discontinuous games, Econometrica 81, 813-824.

Baye, M.R., Tian, G., Zhou, J., 1993. Characterization of the existence of equilibria in games with discontinuous and non-quasiconcave payoffs. Rev. Econ. Stud. 60, 935-948. 
Baye, M.R., Kovenock, D., de Vries, C.G., 1994. The solution to the Tullock rent-seeking game when $R>2$ : Mixed-strategy equilibria and mean dissipation rates. Public Choice 81, 363-380.

Baye, M.R., Kovenock, D., de Vries, C.G., 1996. The all-pay auction with complete information. Econ. Theory 8, 291-305.

Bich, P., 2009. Existence of pure Nash equilibria in discontinuous and non quasiconcave games. Int. J. Game Theory 38, 395-410.

Carbonell-Nicolau, O., Ok, E.A., 2007. Voting over income taxation. J. Econ. Theory 134, 249-286.

Carmona, G., 2005. On the existence of equilibria in discontinuous games: three counterexamples. Int. J. Game Theory 33, 181-187.

Carmona, G., 2011. Understanding some recent existence results for discontinuous games. Econ. Theory 48, 31-45.

de Castro, L.I., 2011. Equilibria existence in regular discontinuous games, Econ. Theory 48, 67-85.

Dasgupta, P., Maskin, E., 1986. The existence of equilibrium in discontinuous economic games, I: Theory. Rev. Econ. Stud. 53, 1-26.

Ding, X.P., Park, J.Y., 2002. Continuous selection theorem, coincidence theorem, and generalized equilibrium in $L$-convex spaces. Comput. Math. Appl. 44, 95-103.

Duggan, J., 2007. Equilibrium existence for zero-sum games and spatial models of elections. Games and Econ. Behav. 60, 52-74.

Glicksberg, I.L., 1952. A Further generalization of the Kakutani fixed point theorem with applications to Nash equilibrium points. Proc. Amer. Math. Soc. 38, 170-174

Glycopantis, D., Muir, A., 2004. The compactness of $\operatorname{Pr}(K)$. Adv. Math. Econ. 6, 39-53.

Kechris A.S., 1995. Classical Descriptive Set Theory, New York: SpringerVerlag.

Klose, B., Kovenock, D., 2013. The all-pay auction with complete in- 
formation and identity-dependent externalities. Working paper No. 118, University of Zurich.

Lan, K.Q., Wu, J.H., 2002. A fixed-point theorem and applications to problems on sets with convex sections and to Nash equilibria. Math. Comput. Model 36, 139-145.

McLennan, A., Monteiro, P.K., Tourky, R., 2011. Games with discontinuous payoffs: a strengthening of Reny's existence theorem. Econometrica 79, 1643-1664.

Monteiro, P.K., Page, F.H., 2007. Uniform payoff security and Nash equilibrium in compact games. J. Econ. Theory 134, 566-575.

Page, F.H., Monteiro, P.K., 2003. Three principles of competitive nonlinear pricing. J. Math. Econ. 39, 63-109.

Prokopovych, P., 2011. On equilibrium existence in payoff secure games. Econ. Theory 48, 5-16.

Prokopovych, P., 2013. The single deviation property in games with discontinuous payoffs. Econ. Theory 53, 383-402.

Reny, P.J., 1999. On the existence of pure and mixed strategy Nash equilibria in discontinuous games. Econometrica 67, 1029-1056.

Reny, P.J., 2009. Further results on the existence of Nash equilibria in discontinuous games. Working paper, University of Chicago.

Reny, P.J., 2011. Strategic approximation of discontinuous games. Econ. Theory 48, 17-29.

Reny, P.J., 2013. Nash equilibrium in discontinuous games. Working paper, University of Chicago.

Siegel, R., 2009. All-pay contests. Econometrica 77, 71-92.

Simon, L., 1987. Games with discontinuous payoffs. Rev. Econ. Stud. 54, 569-597.

Simon, L.K., Zame, W.R., 1990. Discontinuous games and endogenous sharing rules. Econometrica 58, 861-872.

Sion, M., Wolfe, P., 1957. On a game without a value, in: Contributions 
to the Theory of Games, III, Princeton. Ann. Math. Stud. 39, 299-306.

Tian, G., 1992. Generalizations of the FKKM theorem and the Ky Fan minimax Inequality, with applications to maximal elements, price equilibrium, and complementarity. J. Math. Anal. Appl. 170, 457-471.

Tullock, G., 1980. Efficient rent-seeking, In: J.M. Buchanan et al. (Eds) Toward a Theory of the Rent-Seeking Society, Texas A\&M University Press, College Station.

Yannelis, N.C., Prabhakar, N.D., 1983. Existence of maximal elements and equilibria in linear topological spaces. J. Math. Econ. 12, 233-245.

Yannelis, N.C., 1991. The core of an economy without ordered preferences, In: Khan,M.A., Yannelis, N.C. (eds.) Equilibrium Theory in Infinite Dimensional Spaces. Springer, Berlin. 